\title{
Periodic event-triggered control for nonlinear networked control systems
}

\author{
W. Wang, R. Postoyan, D. Nešić and W.P.M.H. Heemels
}

\begin{abstract}
Periodic event-triggered control (PETC) is an appealing paradigm for the implementation of controllers on platforms with limited communication resources, a typical example being networked control systems. In PETC, transmissions over the communication channel are triggered by an event generator, which depends solely on the available plant and controller data, and is only evaluated at given sampling instants to enable its digital implementation. In this paper, we consider the general scenario where the controller communicates with the plant via multiple decoupled networks. Each network may contain multiple nodes, in which case a dedicated protocol is used to schedule transmissions among these nodes. The transmission instants over the networks are asynchronous and generated by local event generators. At given sampling instants, the local event generator evaluates a rule, which only involves the measurements and the control inputs available locally, to decide whether a transmission is needed over the considered network. Following the emulation approach, we show how to design the local triggering generators to ensure input-to-state stability and $\mathcal{L}_{p}$-stability for the overall system based on a continuous-time output feedback controller that robustly stabilizes the network-free system. The method is applied to a class of Lipschitz nonlinear systems, for which we formulate the design conditions as linear matrix inequalities. The effectiveness of the scheme is illustrated via simulations of a nonlinear example.
\end{abstract}

\section{INTRODUCTION}

$\mathbf{N}$ Etworked control systems (NCS) refer to systems in which the plant and the controller communicate via networks. Integrating networks into control systems, compared with the traditional dedicated point-to-point (wired) links, has major advantages like lower cost, reduced weight and power, simpler installation and maintenance, and higher reliability [1]. Moreover, the NCS configuration is essential when the plant consists of many subsystems, which are physically distributed and interconnected to coordinate their tasks and achieve an overall objective, see their applications in smart grids, widearea systems or for systems with distributed sensors, actuators and controllers. A major challenge in NCS is to design control strategies which do not "overuse" the network, to limit the

This work is supported by the Australian Research Council under the Discovery Project DP170104099, the ANR under the grant HANDY (ANR18-CE40-0010-02), and the Innovational Research Incentives Scheme under the VICI grant "Wireless control systems: A new frontier in automation" (No. 11382) awarded by NWO (The Netherlands Organisation for Scientific Research) and STW (Dutch Technology Foundation).

W. Wang and D. Nešić are with the Department of Electrical and Electronic Engineering, The University of Melbourne, Parkville, 3010, Victoria, Australia wei.wang@unimelb.edu.au, dnesiccunimelb.edu.au

R. Postoyan is with the Université de Lorraine, CNRS, CRAN, F-54000 Nancy, France romain.postoyaneuniv-lorraine.fr

W.P.M.H. Heemels is with the Department of Mechanical Engineering, Eindhoven University of Technology, Eindhoven, The Netherlands, m. heemelsatue.nl transmission delays and the occurrence of packet losses, which may destroy the desired closed-loop system properties. An attractive approach in this context is event-triggered control, which adapts the transmission instants based on the current state, input and/or output measurement of the plant, see [2] and the references therein. The idea of ETC is to use the network only when this is needed by generating transmissions whenever a state or output-dependent condition is satisfied. Most literature on ETC focuses on continuous event-triggered control (CETC), in the sense that the triggering condition is evaluated at all times, see, for instance, [3]-[7]. Although CETC may significantly reduce the number of transmissions compared with traditional periodic sampling, the continuous evaluation of the triggering condition causes issues when sensors are battery powered for mobility and/or flexibility reasons. Moreover, it is not even possible to evaluate triggering rules continuously when the implementation platform is digital. In this case, it is more natural to evaluate the triggering criterion at some discrete sampling instants, leading to periodic eventtriggered control (PETC), see [8], [9].

Hybrid systems are commonly used to model CETC systems (e.g. [4], [7], [10], [11]), as the plant and the controller are often described by continuous-time systems and transmissions are discrete events, which can be modeled by jumps. The generic results in [12] about the sampled-and-hold implementations of hybrid controllers ensure that the emulation of a continuous event-triggered controller as a periodic eventtriggered controller still "works", if the sampling period is sufficiently small. To be more precise, the uniform global asymptotic stability of a compact set ensured by CETC is semiglobally and practically preserved for fast sampling by PETC. Unfortunately, these results do not provide exploitable explicit bounds on the sampling period. Furthermore, it is of interest to preserve global asymptotic stability properties in PETC, instead of semiglobal practical asymptotic stability. Works addressing these points have mostly been developed for systems with linear dynamics, see [8], [13]-[16]. On the other hand, PETC results for nonlinear systems are scarce. In [9, Chapter 6.5] and [17], it is explained how to convert general continuous state-feedback event-triggered controllers to periodic event-triggered ones, while (approximately) preserving the properties of the former. The work in [18] develops observer-based output-feedback controllers for a class of nonlinear Lipschitz systems and a practical stability property is ensured at the end. Another work is [19], where output-feedback PETC scheme is studied to ensure global asymptotic stability for a class of polynomial nonlinear systems. Obviously, PETC for nonlinear systems is at its early stage and a lot remains to 
be done. In particular, there is a need for systematic design frameworks, which are flexible enough to cope with output feedbacks as well as exogenous disturbances. The primary aim of this paper is to address this challenge.

We study plants modeled by a continuous-time nonlinear system affected by exogenous disturbances and for which only some output is available for control. We proceed by emulation to design the periodic event-triggered controller. Thus, we first assume that we know an output feedback controller, which robustly stabilizes the plant in absence of communication constraints, in the sense that it either ensures an input-to-state stability or a $\mathcal{L}_{p}$ stability property for the closed-loop system with respect to the exogenous disturbances as well as output and input noises. At this stage, any continuous-time design technique can be applied. We then implement the controller over networks. We investigate the scenario where multiple asynchronously operating networks are used to connect the controller to the plant: this is an additional novelty of this work. This setup is relevant, for instance, when one network ensures the communication from the sensors to the controller, and another one is used to connect the controller to the actuators. The sensors and the actuators are grouped into nodes, which are connected to a given network. The transmissions over each network are generated by a local triggering generator. The latter collects measurements and control inputs, which are locally available, at some sampling instants specific to the considered network (and not necessarily periodic), it evaluates a criterion and then decides whether a node needs to transmit its packet over this network. The transmitting node is selected according to the local scheduling rule, such as the Round-Robin (RR) or Try-Once-Discard (TOD) protocol considered in [1], [20]. To design the local triggering generators, we therefore have to define three elements: (i) the criterion; (ii) the sampling instants at which the criterion is evaluated; (iii) the scheduling rule. Regarding the scheduling rules, we require that they are uniformly globally asymptotically stable as characterized in [20], which cover the RR, TOD and the sampled-data protocols. We also make assumptions on the robust stability of the original closed-loop system in the absence of network, which can be checked a priori. Note that imposing robust stability properties is required for any nonlinear control systems to be implementable in practice. Based on these assumptions, we provide the expression of the local triggering conditions as well as an explicit bound on the maximum allowable sampling periods (MASP), which are used to characterize the sampling instants. We actually show that there is a tradeoff between the MASP of each triggering generator and a parameter used to define the corresponding triggering condition.

The overall system is modeled as a hybrid system using the formalism of [21], [22], for which a jump corresponds to a sampling instant of one local triggering generator. We then ensure an input-to-state stability or a $\mathcal{L}_{p}$-stability with respect to the exogenous disturbances, depending on the assumptions. These results lead to a uniform global asymptotic stability property in the absence of disturbances. The analysis relies on a novel hybrid Lyapunov function. We apply the results to a class of globally Lipschitz nonlinear systems and formulate the assumptions as linear matrix inequalites (LMIs). The obtained LMIs are always verified in the special cases when the nonlinearity only involves the measured output or for any stabilizable and detectable LTI systems. The latter case appears to be a contribution in its own right as it extends the centralized and state-feedback PETC for linear systems in [8], [13] and the output-feedback PETC in [14] to decentralized implementations. Simulation results on a nonlinear system, which is not globally Lipschitz, are also provided.

The decentralized setup we investigate is similar to the one in [4] where continuous event-triggered controllers are synthesized. The fact that we consider PETC, as opposed to CETC, benefits for digital implementations, which leads to additional difficulties. Because the triggering rules are continuously evaluated in CETC, properties, which are essential to guarantee stability, are ensured at all times. This is no longer the case in PETC, as the triggering criteria are only checked at some sampling instants and may therefore be violated between two successive sampling instants. As a result, our approach requires a new hybrid model, a different set of assumptions as well as a novel hybrid Lyapunov function compared to [4]. Note that CETC as proposed in [4] relies on timeregularization, as the triggering criterion is evaluated continuously after a fixed waiting time has elapsed since the previous event. This is different from PETC as done in the paper, as the triggering conditions here are evaluated only at some sampling instants, which facilitates digital implementations. Compared to [9, Chapter 6.5] and [17], the results are applicable for decentralized output-feedback control, tolerate the presence of exogenous disturbances and explicitly reveal a link between the triggering conditions and the sampling instants. Compared to [18], we consider exogenous disturbances, a decentralized scenario, we do not restrict our attention to nonlinear systems with a specific structure, and we ensure asymptotic stability in the absence of perturbations. Conference versions of this work can be found in [23] and [24]. In particular, a centralized fullstate feedback PETC is provided for disturbance-free systems in [23], and a centralized output-feedback control for systems implemented on a single network is studied in [24] where only input-to-state stability results are provided.

To summarize, our work leads to the following contributions on PETC: (i) a generic design framework of the triggering generators for nonlinear systems, which is applicable for output feedback control as well as in the presence of exogenous disturbances; (ii) decentralized PETC strategies over multiple asynchronously operating networks, for the first time to the best of our knowledge; (iii) a novel hybrid Lyapunov function is constructed to investigate stability properties of the system; (iv) even in the particular case of linear systems, the results extend those in [8], [13], [14] to decentralized output-feedback control.

The rest of the paper is organized as follows. The notation and preliminaries on hybrid systems are given in Section II. We state the problem and present the hybrid model in Section III. The main results are provided in Section IV and applied to a class of globally Lipschitz nonlinear systems in Section V. Simulation results for a nonlinear system are given in Section VI and conclusions are provided in Section VII. The proofs 
are postponed to the appendix, where technical lemmas are also provided.

\section{PRELIMINARIES}

Let $\mathbb{Z}_{>0}:=\{1,2, \ldots\}, \mathbb{Z}_{\geq 0}:=\{0,1,2, \ldots\}, \mathbb{R}:=$ $(-\infty, \infty)$ and $\mathbb{R}_{\geq 0}:=[0, \infty)$. For $k_{0} \in \mathbb{Z}_{\geq 0}$ and $\Gamma \subset \mathbb{Z}_{\geq 0}$, $k_{0}+\Gamma:=\left\{k_{0}+k: k \in \Gamma\right\}$. For sets $A$ and $B$ in a universe $U, A \backslash B:=\{x \in U: x \in A$ and $x \notin B\}$. Let $\mathbf{0}_{n}$ and $\mathbf{1}_{n}, n \in \mathbb{Z}_{>0}$, be the $n$-dimensional vector for which elements are all zeros and ones, respectively. Let $\mathbf{0}_{n \times n}$ and $\mathbf{I}_{n \times n}$ be the square zero matrix and the identity matrix of dimension $n$, respectively. Let $|x|$ denote the Euclidean norm of the vector $x \in \mathbb{R}^{n}$. Let $\lambda_{\min }(P)$ and $\lambda_{\max }(P)$ stand for the minimum and maximum eigenvalues of real symmetric matrix $P$, respectively. For $x \in \mathbb{R}^{n}$ and $y \in \mathbb{R}^{m},(x, y)$ stands for $\left[x^{T}, y^{T}\right]^{T}$. Given a set $\mathcal{A} \subset \mathbb{R}^{n}$ and $x \in \mathbb{R}^{n}$, we define the distance of $x$ to $\mathcal{A}$ as $|x|_{\mathcal{A}}:=\inf _{y \in \mathcal{A}}|x-y|$. A set-valued mapping $M: \mathbb{R}^{m} \rightrightarrows \mathbb{R}^{n}$ is outer semi-continuous when its graph $\left\{(y, z) \in \mathbb{R}^{m} \times \mathbb{R}^{n}: z \in M(y)\right\}$ is closed, see Lemma 5.10 in [22]. A function $\gamma: \mathbb{R}_{\geq 0} \rightarrow \mathbb{R}_{\geq 0}$ is of class- $\mathcal{K}$, if it is continuous, zero at zero and strictly increasing and it is of class- $\mathcal{K}_{\infty}$ if, in addition, it is unbounded. A function $\gamma: \mathbb{R}_{\geq 0} \times \mathbb{R}_{\geq 0} \rightarrow \mathbb{R}_{\geq 0}$ is of class- $\mathcal{K} \mathcal{L}$, if it is continuous, $\gamma(\cdot, r)$ is of class- $\mathcal{K}$ for each $r \in \mathbb{R}_{\geq 0}$, and, for each $s \in \mathbb{R}_{\geq 0}$, $\gamma(s, \cdot)$ is decreasing to zero. The notation $\left(t^{\prime}, j^{\prime}\right) \leq(t, j)$ refers to $t^{\prime} \leq t$ and $j^{\prime} \leq j$ with $t, t^{\prime} \in \mathbb{R}_{\geq 0}$ and $j, j^{\prime} \in \mathbb{Z}_{\geq 0}$. For $x, v \in \mathbb{R}^{n}$ and locally Lipschitz $U: \mathbb{R}^{n} \rightarrow \mathbb{R}, U^{\circ}(x ; v)$ is the Clarke derivative of the function $U$ at $x$ in the direction $v$, i.e. $U^{\circ}(x ; v):=\limsup _{y \rightarrow x, \lambda \downarrow 0} \frac{U(y+\lambda v)-U(y)}{\lambda}$. This notion will be useful as we will be working with locally Lipschitz Lyapunov functions, which are not differentiable everywhere. We omit the definitions on hybrid systems and refer the reader to [21], [22].

\section{PETC SETUP AND HYBRID MODEL}

In this section, we introduce the setup and model the overall system as a hybrid system. We then formally state the problem.

\section{A. PETC setup}

We consider the plant model

$$
\begin{aligned}
\dot{x}_{p} & =f_{p}\left(x_{p}, u, w\right) \\
y & =g_{p}\left(x_{p}\right),
\end{aligned}
$$

where $x_{p} \in \mathbb{R}^{n_{p}}$ is the state, $w \in \mathbb{R}^{n_{w}}$ is the exogenous disturbance, $u \in \mathbb{R}^{n_{u}}$ is the control input, and $y \in \mathbb{R}^{n_{y}}$ is the plant output. As already mentioned in the introduction, we use an emulation-based design approach. We therefore assume that we know an output-feedback controller

$$
\begin{aligned}
\dot{x}_{c} & =f_{c}\left(x_{c}, y\right) \\
u & =g_{c}\left(x_{c}\right)
\end{aligned}
$$

with state $x_{c} \in \mathbb{R}^{n_{c}}$, which robustly stabilizes the origin of (1) in a sense made precise in Section IV-A. The functions $f_{p}$ and $f_{c}$ are assumed to be continuous, and $g_{p}$ and $g_{c}$ are assumed to be continuously differentiable and zero at zero.
Any controller design method can be used to obtain controller (2), such as backstepping, forwarding, feedback linearization, high-gain techniques etc.

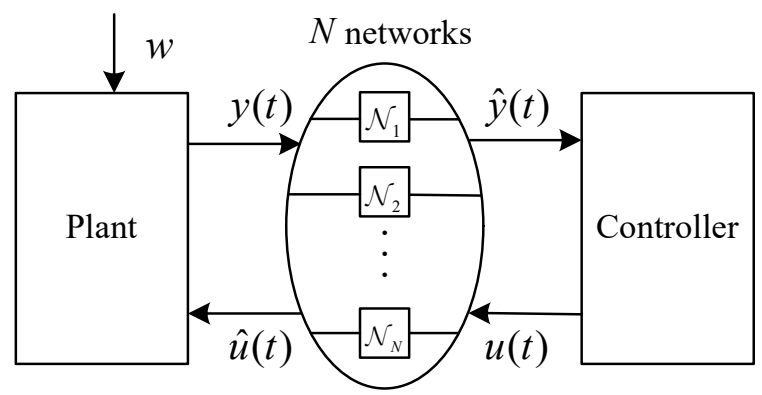

Fig. 1: Block diagram of the setup

We consider the scenario where plant (1) and controller (2) communicate with each other via multiple networks, as illustrated in Figure 1. In particular, sensors and actuators are connected by $N \in \mathbb{Z}_{>0}$ independently and asynchronously operating networks $\mathcal{N}_{1}, \ldots, \mathcal{N}_{N}$. Let $\bar{N}:=\{1,2, \ldots, N\}$ and $v:=(y, u) \in \mathbb{R}^{n_{y}+n_{u}}$. For simplicity of exposition, we assume $v=\left(v_{1}, \ldots, v_{N}\right)$ (after reordering, if necessary), where $v_{i}, i \in \bar{N}$, corresponds to the sensors and the actuators whose signals are transmitted through network $\mathcal{N}_{i}$.

A local event-triggering generator generates the sequence of transmission instants for each network $\mathcal{N}_{i}, i \in \bar{N}$, in the following manner. A triggering condition is evaluated at each sampling instant $s_{j}^{i}, i \in \bar{N}, j \in \mathbb{Z}_{\geq 0}$, where

$$
\varepsilon_{i} \leq s_{j+1}^{i}-s_{j}^{i} \leq T_{i}
$$

with $T_{i}>0$ the upper bound on the inter-sampling times and $\varepsilon_{i} \in\left(0, T_{i}\right]$ the minimum time between two successive evaluations of the triggering condition. Note that each network has its own sequence of sampling instants, which is not necessarily periodic or synchronized with the other networks. Consequently, the sequence of transmission instants of network $\mathcal{N}_{i}$, which we denote $\left\{t_{\kappa_{i}}^{i}\right\}_{\kappa_{i} \in \mathbb{Z}_{\geq 0}}$, is a subsequence of $\left\{s_{j}^{i}\right\}_{j \in \mathbb{Z}_{\geq 0}}$, and two successive transmissions are spaced by at least $\varepsilon_{i}$ units of time in view of (3), thereby avoiding the Zeno phenomenon. Parameter $\varepsilon_{i}$ reflects the minimum achievable transmission interval given by the hardware constraints. Note that $\varepsilon_{i}$ can be chosen arbitrarily in the set $\left(0, T_{i}\right]$. In fact, the stability and performance results below apply for any $\varepsilon_{i} \in\left(0, T_{i}\right]$. In practical, $\varepsilon_{i}>0$ is determined by the hardware constraint. We assume that transmission delays and quantization effects are negligible. Each transmission generator consists of a triggering law and a scheduling rule. We need to introduce some variables before presenting those.

We denote by $\hat{u}$ the networked version of $u$ available to plant (1). Similarly, controller (2) has access to $\hat{y}$, the networked version of $y$. We let $\hat{v}$ be the networked version of $v$ and we partition it as $\left(\hat{v}_{1}, \ldots, \hat{v}_{N}\right)$ in the same way as $v$ is. Thus, $\hat{v}_{i}$, $i \in \bar{N}$, is related to the network $\mathcal{N}_{i}$. Between two successive transmission instants, $\hat{v}_{i}$ is governed by

$\dot{\hat{v}}_{i}=\hat{f}_{v_{i}}\left(\hat{v}, g_{p}\left(x_{p}\right), g_{c}\left(x_{c}\right)\right), t \in\left(s_{j}^{i}, s_{j+1}^{i}\right), j \in \mathbb{Z}_{\geq 0}, i \in \bar{N}$, 
where $\hat{f}_{v_{i}}$ is the holding function corresponding to network $\mathcal{N}_{i}$ and we define $\hat{f}_{v}:=\left(\hat{f}_{v_{1}}, \ldots, \hat{f}_{v_{N}}\right)$. Zero-order-hold devices correspond to $\hat{f}_{v}=0$ for instance. Other holding functions can also be envisioned, like model-based ones, see, for example, [5]. Before modeling the dynamics of $\hat{v}_{i}, i \in \bar{N}$, at each sampling instant $s_{j}^{i}$, we introduce the vector of networkinduced errors $e_{i}:=\hat{v}_{i}-v_{i} \in \mathbb{R}^{n_{e_{i}}}$, where $n_{e_{i}} \in \mathbb{Z}_{>0}$ satisfies $\sum_{i=1}^{N} n_{e_{i}}=n_{y}+n_{u}$. Hence, $n_{e_{i}}$ is the number of sensor/actuator signals associated with network $\mathcal{N}_{i}$.

At each sampling instant $s_{j}^{i}, j \in \mathbb{Z}_{\geq 0}$ and $i \in \bar{N}$, a function $\Upsilon_{i}: \mathbb{R}^{n_{e_{i}}} \times \mathbb{R}^{n_{e_{i}}} \times \mathbb{Z}_{\geq 0} \rightarrow \mathbb{R}$ is evaluated, which depends on $v_{i}, \hat{v}_{i}$ and an auxiliary variable $\kappa_{i}$, which counts the number of transmissions over network $\mathcal{N}_{i}$. The expression of $\Upsilon_{i}$ will be given in Section IV-B. A transmission is triggered depending on the sign of $\Upsilon_{i}$, which leads to the update law for $\hat{v}_{i}$ given by

$$
\hat{v}_{i}\left(s_{j}^{i+}\right) \in\left\{\begin{array}{c}
\left\{v_{i}\left(s_{j}^{i}\right)+\chi_{i}\left(e_{i}\left(s_{j}^{i}\right), \kappa_{i}\left(s_{j}^{i}\right)\right)\right\} \\
\text { when } \left.\Upsilon_{i}\left(e_{i}\left(s_{j}^{i}\right), v_{i}\left(s_{j}^{i}\right), \kappa_{i}\left(s_{j}^{i}\right)\right)\right)>0 \\
\left\{\hat{v}_{i}\left(s_{j}^{i}\right)\right\} \\
\text { when } \Upsilon_{i}\left(e_{i}\left(s_{j}^{i}\right), v_{i}\left(s_{j}^{i}\right), \kappa_{i}\left(s_{j}^{i}\right)\right)<0 \\
\left\{\hat{v}\left(s_{j}^{i}\right), v_{i}\left(s_{j}^{i}\right)+\chi_{i}\left(e_{i}\left(s_{j}^{i}\right), \kappa_{i}\left(s_{j}^{i}\right)\right)\right\} \\
\text { when } \Upsilon_{i}\left(e_{i}\left(s_{j}^{i}\right), v_{i}\left(s_{j}^{i}\right), \kappa_{i}\left(s_{j}^{i}\right)\right)=0,
\end{array}\right.
$$

where $\chi_{i}$ models the scheduling protocol corresponding to network $\mathcal{N}_{i}$, such as the $\mathrm{RR}^{1}$, or TOD protocol ${ }^{2}$, or the socalled sampled-data protocol for which $\chi_{i}=0$ when the network is composed of a single node. Expressions of $\chi_{i}$ for various protocols are available in [20], [25] and the cases of RR and TOD are provided next for completeness.

Example 1 (TOD protocol): Let $i \in \bar{N}$ and $\ell_{i} \in \mathbb{Z}_{>0}$ denote the number of nodes of $\mathcal{N}_{i}$ network. TOD protocol is modelled as $\chi_{i}\left(e_{i}\right):=\left(I-\widetilde{\Psi}_{i}\left(e_{i}\right)\right) e_{i}$, where $\widetilde{\Psi}_{i}\left(e_{i}\right):=$ $\operatorname{diag}\left\{\widetilde{\psi}_{1}^{i}\left(e_{i}\right) \mathbf{I}_{n_{1}^{i} \times n_{1}^{i}}, \widetilde{\psi}_{2}^{i}\left(e_{i}\right) \mathbf{I}_{n_{2}^{i} \times n_{2}^{i}}, \ldots, \widetilde{\psi}_{\ell_{i}}^{i}\left(e_{i}\right) \mathbf{I}_{n_{\ell_{i}}^{i} \times n_{\ell_{i}}^{i}}\right\}$.

The functions $\widetilde{\psi}_{s}^{i}$ satisfy $\widetilde{\psi}_{s}^{i}\left(e_{i}\right)=1$ when $s=\min \left(\arg \max _{j \in\left\{1, \ldots, \ell_{i}\right\}}\left|e_{j}^{i}\right|\right)$ and $\widetilde{\psi}_{s}\left(e_{i}\right)=0$ otherwise, for $s \in\left\{1,2, \ldots, \ell_{i}\right\}$.

Example 2 (RR protocol): $\mathrm{RR}$ protocol has the form of $\chi_{i}\left(e_{i}, \kappa_{i}\right):=\left(I-\Delta_{i}\left(\kappa_{i}\right)\right) e_{i}$, where $\Delta_{i}\left(\kappa_{i}\right)=$ $\operatorname{diag}\left\{\delta_{1}^{i}\left(\kappa_{i}\right) \mathbf{I}_{n_{1}^{i} \times n_{1}^{i}}, \delta_{2}^{i}\left(\kappa_{i}\right) \mathbf{I}_{n_{2}^{i} \times n_{2}^{i}}, \ldots, \delta_{\ell_{i}}^{i}\left(\kappa_{i}\right) \mathbf{I}_{n_{\ell_{i}}^{i} \times n_{\ell_{i}}^{i}}\right\}$, $\delta_{s}^{i}\left(\kappa_{i}\right)=1$ for $s \in\left\{1,2, \ldots, \ell_{i}\right\}$ when $s-1=\kappa_{i} \bmod \ell_{i}$ and $\delta_{s}^{i}\left(\kappa_{i}\right)=0$ otherwise.

Let $\ell_{i} \in \mathbb{Z}_{>0}$ be the number of nodes of network $\mathcal{N}_{i}$, and $v_{i}$ and $\hat{v}_{i}$ are, respectively, partitioned as $\left(v_{i, 1}, \ldots, v_{i, \ell_{i}}\right)$, and $\left(\hat{v}_{i, 1}, \ldots, \hat{v}_{i, \ell_{i}}\right)$ (after reordering these, if needed), where $v_{i, j}$ and $\hat{v}_{i, j}, j \in\left\{1, \cdots, \ell_{i}\right\}$, denote a group of sensors or/and actuators associated to the same node on $\mathcal{N}_{i}$. In view of (5), when $\Upsilon_{i}\left(e_{i}\left(s_{j}^{i}\right), v_{i}\left(s_{j}^{i}\right), \kappa_{i}\left(s_{j}^{i}\right)\right)>0$, a transmission occurs over network $\mathcal{N}_{i}$ at time $s_{j}^{i}$ and the scheduling protocol grants access to the network to a single node, say the $k$-th

\footnotetext{
${ }^{1} \mathrm{RR}$ protocol assigns access to network in a predetermined and cyclic manner.

${ }^{2}$ TOD protocol gives access to the node with the largest mismatch between the current signal value and the last transmitted one.
}

node with $k \in\left\{1,2, \ldots, \ell_{i}\right\}$. Then, $\hat{v}_{i, k}\left(s_{j}^{i+}\right)=v_{i, k}\left(s_{j}^{i}\right)$ and $\hat{v}_{i, m}\left(s_{j}^{i+}\right)=\hat{v}_{i, m}\left(s_{j}^{i}\right)$ for all $m \in\left\{1,2, \ldots, \ell_{i}\right\} \backslash\{k\}$. When $\Upsilon_{i}\left(e_{i}\left(s_{j}^{i}\right), v_{i}\left(s_{j}^{i}\right), \kappa_{i}\left(s_{j}^{i}\right)\right)<0$, no transmission occurs, $\kappa_{i}$ and the complete vector $\hat{v}_{i}$ remain unchanged. When $\Upsilon_{i}\left(e_{i}\left(s_{j}^{i}\right), v_{i}\left(s_{j}^{i}\right), \kappa_{i}\left(s_{j}^{i}\right)\right)=0$, the model allows two possibilities: either a transmission occurs or not. This construction ensures that the jump map in (5) is outer semi-continuous, which is essential for the hybrid model presented below to be (nominally) well-posed, see Chapter 6 in [22] for more details. Note that the transmissions over the $N$ networks are independently generated, as a result, several transmissions can occur at the same time, but over distinct networks.

We are almost ready to model the overall system. Before that, we need to write the dynamics of the network-induced errors. Let $x:=\left(x_{p}, x_{c}\right) \in \mathbb{R}^{n_{x}}$ and $n_{x}:=n_{p}+n_{c}$. We deduce from (5) that the variable $e_{i}$ has the following dynamics at jumps

$$
e_{i}\left(s_{j}^{i+}\right) \in h_{i}\left(x\left(s_{j}^{i}\right), e_{i}\left(s_{j}^{i}\right), \kappa_{i}\left(s_{j}^{i}\right)\right),
$$

where

$h_{i}\left(x, e_{i}, \kappa_{i}\right):=\left(1-\Gamma_{i}\left(e_{i}, v_{i}, \kappa_{i}\right)\right) e_{i}+\Gamma_{i}\left(e_{i}, v_{i}, \kappa_{i}\right) \chi_{i}\left(e_{i}, \kappa_{i}\right)$,

and $\Gamma_{i}: \mathbb{R}^{n_{e_{i}}} \times \mathbb{R}^{n_{e_{i}}} \times \mathbb{Z}_{\geq 0} \rightrightarrows\{0,1\}$ in (7) indicates whether a transmission occurs. Based on the discussion above (5), $\Gamma_{i}\left(e_{i}, v_{i}, \kappa_{i}\right)=\{1\}$ when $\Upsilon_{i}\left(e_{i}, v_{i}, \kappa_{i}\right)>0$, which corresponds to a transmission and $h_{i}\left(x, e_{i}, \kappa_{i}\right)=\chi_{i}\left(e_{i}, \kappa_{i}\right)$ in this case. When $\Upsilon_{i}\left(e_{i}, v_{i}, \kappa_{i}\right)<0, \Gamma_{i}\left(e_{i}, v_{i}, \kappa_{i}\right)=\{0\}$ and this corresponds to no transmission and $h_{i}\left(x, e_{i}, \kappa_{i}\right)=e_{i}$. When $\Upsilon_{i}\left(e_{i}, v_{i}, \kappa_{i}\right)=0, \Gamma_{i}\left(e_{i}, v_{i}, \kappa_{i}\right)=\{0,1\}$ covers the above two possibilities. In agreement with [20], we call (6) the protocol map. We see from the right hand-side of (7) that $h_{i}$ depends on $v_{i}$ and not on the complete vector of $x$. Writing

$$
v_{i}=g_{v_{i}}(x),
$$

since $v_{i}$ is composed of components of $y$ and $u$, which depend on $x$ in view of (1) and (2), we make $h_{i}$ depend on $x$ and not $v_{i}$ in (7), for the sake of convenience. We note that $h_{i}$ depends on the state $x$ contrary to [20], [26], [27], which will have important consequences on the stability property and analysis of the protocols compared to the latter references, see Remark 3 in Section IV-B.

\section{B. Hybrid model}

We model the overall system as a hybrid system using the formalism of [21], [22] so that we can resort to the analytical tools of [21], [22] to study the stability properties of the system. We introduce for this purpose clock variables $\tau_{i} \in \mathbb{R}_{\geq 0}$ for $i \in \bar{N}$ to keep track of the time elapsed since the last evaluation of the triggering criterion of network $\mathcal{N}_{i}$. Thus, $\tau_{i}$ and $\kappa_{i}$, the transmission counter of network $\mathcal{N}_{i}$, have the dynamics

$$
\begin{aligned}
& \left\{\begin{array}{l}
\dot{\tau}_{i}=1 \\
\dot{\kappa}_{i}=0
\end{array}\right. \\
& \left\{\begin{array}{l}
\tau_{i}^{+}=0 \\
\kappa_{i}^{+} \in \kappa_{i}+\Gamma_{i}\left(e_{i}, v_{i}, \kappa_{i}\right)
\end{array}\right.
\end{aligned}
$$


where $\Gamma_{i}$ is introduced after (7). Let $\tau:=\left(\tau_{1}, \ldots, \tau_{N}\right)$ and $\kappa:=\left(\kappa_{1}, \ldots, \kappa_{N}\right)$. We model the overall closed-loop system as

$$
\begin{array}{rlrl}
\dot{q} & =F(q, w) & & q \in C \\
q^{+} \in G(q) & & q \in D,
\end{array}
$$

where $q:=(x, e, \kappa, \tau) \in \mathcal{X}:=\mathbb{R}^{n_{x}} \times \mathbb{R}^{n_{e}} \times \mathbb{Z}^{N} \times \mathbb{R}^{N}$,

$$
\begin{aligned}
C:= & \mathbb{R}^{n_{x}} \times \mathbb{R}^{n_{e}} \times \mathbb{Z}_{\geq 0}^{N} \times \mathbb{T}_{1} \times \ldots \times \mathbb{T}_{N} \\
D:= & \bigcup_{i=1}^{N} D_{i} \\
D_{i}:= & \mathbb{R}^{n_{x}} \times \mathbb{R}^{n_{e}} \times \mathbb{Z}_{\geq 0}^{N} \times \mathbb{T}_{1} \times \ldots \times \mathbb{T}_{i-1} \times\left[\varepsilon_{i}, T_{i}\right] \\
& \times \mathbb{T}_{i+1} \times \ldots \times \mathbb{T}_{N},
\end{aligned}
$$

and $\mathbb{T}_{i}:=\left[0, T_{i}\right]$. The mapping $F$ in (9) is defined as, for $q \in C, F(q, w):=\left(f(x, e, w), g(x, e, w), \mathbf{0}_{N}, \mathbf{1}_{N}\right)$, where $f(x, e, w):=\left(f_{p}\left(x_{p}, g_{c}\left(x_{c}\right)+e_{u}, w\right), f_{c}\left(x_{c}, g_{p}\left(x_{p}\right)+e_{y}\right)\right)$, $g(x, e, w):=\left(g_{1}(x, e, w), \ldots, g_{N}(x, e, w)\right)$, and for $i \in \bar{N}$,

$$
\begin{aligned}
g_{i}(x, e, w) & :=\hat{f}_{v_{i}}\left(g_{v_{i}}(x)+e_{i}, g_{v_{i}}(x)\right)-f_{v_{i}}(x, e, w) \\
f_{v_{i}}(x, e, q) & :=\frac{\partial g_{v_{i}}}{\partial x} f(x, e, w),
\end{aligned}
$$

with $f_{p}, g_{p}, f_{c}, g_{c}, \hat{f}_{v_{i}}, g_{v_{i}}$ coming from (1), (2), (4) and (8), respectively. The set-valued mapping $G$ is defined, for $q \in \mathcal{X}$, as $G(q):=\bigcup_{i=1}^{N} \mathcal{G}_{i}(q)$ with

$$
\begin{aligned}
& \mathcal{G}_{i}(q):= \\
& \left\{\left\{\left(\begin{array}{c}
x \\
\mathcal{H}_{i}(x, e, \kappa) \\
\kappa+\Gamma_{i}\left(e_{i}, x, \kappa_{i}\right) \bar{\Lambda}_{i} \\
\Lambda_{i} \tau
\end{array}\right)\right\} \quad \text { when } q \in D_{i}\right. \\
& \emptyset
\end{aligned}
$$

where $\Lambda_{i} \in \mathbb{R}^{N \times N}$ and $\bar{\Lambda}_{i} \in \mathbb{R}^{N \times N}$ are diagonal matrices, for the former the diagonal elements are 1 except the $i$-th one which is 0 , and for the latter the diagonal elements are 0 except the $i$-th one which is 1 . Hence, $\Lambda_{i}+\bar{\Lambda}_{i}=\mathbf{I}_{N \times N}$. Function $\mathcal{H}_{i}: \mathbb{R}^{n_{x}} \times \mathbb{R}^{n_{e}} \times \mathbb{Z}_{>0}^{N} \rightarrow \mathbb{R}^{n_{e}}$ is defined as $\mathcal{H}_{i}(x, e, \kappa):=\left(e_{1}, e_{2}, \ldots, e_{i-1}, h_{i}\left(x, e_{i}, \kappa_{i}\right), e_{i+1}, \ldots, e_{N}\right)$, where $h_{i}$ comes from (7). The map $\mathcal{G}_{i}$ describes how $e$ jumps when a transmission occurs over network $\mathcal{N}_{i}: e_{i}$ is updated to $h_{i}\left(x, e_{i}, \kappa_{i}\right), \kappa_{i}$ is incremented to $\kappa_{i}+1$ when the local generator triggers a transmission, otherwise it keeps the same value, and $\tau_{i}$ is always reset to 0 after a jump. The function $\mathcal{G}_{i}$ keeps $x, e_{j}, \kappa_{j}, \tau_{j}$ unchanged for all $j \in \bar{N} \backslash\{i\}$. In model (9), simultaneous transmissions over different networks are modeled by successive jumps with no flow in between.

\section{Problem statement}

Our objective is to design the local triggering generators, namely $\Upsilon_{i}$ and $T_{i}, i \in \bar{N}$, to ensure either input-to-state (ISS) or $\mathcal{L}_{p}$ stability properties for system (9), as defined next.

Definition 1: Set $\mathcal{S} \subset \mathcal{X}$ is input-to-state stable (ISS) for system (9) if there exist $\beta \in \mathcal{K} \mathcal{L}$ and $\psi \in \mathcal{K}_{\infty}$ such that any solution pair $(\varphi, w)$ satisfies $^{3}|\varphi(t, j)|_{\mathcal{S}} \leq \beta\left(|\varphi(0,0)|_{\mathcal{S}}, t+\right.$

\footnotetext{
${ }^{3}$ See the definition of $\|w\|_{\infty}$ in [21].
}

$j)+\psi\left(\|w\|_{\infty}\right)$ for all $(t, j) \in \operatorname{dom} \varphi$. We say that $\mathcal{S}$ is exponentially-ISS with a linear gain when $\beta\left(s_{1}, s_{2}\right)=$ $k s_{1} \exp \left(-c s_{2}\right)$ and $\psi(s)=\gamma s$ for some $k, c, \gamma>0$ and for $s_{1}, s_{2}, s \geq 0$.

We define $\mathcal{L}_{p}$-stability with respect to output $z:=\eta(x, w)$, which may correspond to $y$ or not.

Definition 2: System (9) is $\mathcal{L}_{p}$-stable from $w$ to $z$ with respect to set $\mathcal{S} \subset \mathcal{X}$ with gain less than or equal to $\theta$, if there exists $\tilde{\gamma} \in \mathcal{K}_{\infty}$ such that any solution pair $(\varphi, w)$ satisfies $^{4}$ $\|z\|_{\mathcal{L}_{p}} \leq \tilde{\gamma}\left(|\varphi(0,0)|_{\mathcal{S}}\right)+\theta\|w\|_{\mathcal{L}_{p}}$.

\section{Main Results}

In this section, we first state the assumption we make on the closed-loop system (1)-(2) and the scheduling rule, based on which we construct the triggering condition $\Upsilon_{i}$ and the bound on $T_{i}$, for $i \in \bar{N}$. We then present the stability guarantees.

\section{A. Assumptions}

We assume that each $e_{i}$-system in (9) satisfies the following properties.

Assumption 1: For each $i \in \bar{N}$, there exist a locally Lipschitz function $W_{i}: \mathbb{R}^{n_{e_{i}}} \times \mathbb{Z}_{\geq 0} \rightarrow \mathbb{R}_{\geq 0}$, a continuous function $\widetilde{H}_{i}: \mathbb{R}^{n_{x}} \times \mathbb{R}^{n_{e}} \times \mathbb{R}^{n_{w}} \rightarrow \mathbb{R}_{\geq 0}, \underline{\alpha}_{W_{i}}, \bar{\alpha}_{W_{i}} \in \mathcal{K}_{\infty}$, $\rho_{i} \in[0,1)$ and $L_{W_{i}} \geq 0$ such that the following hold.

(i) For any $e_{i} \in \mathbb{R}^{n_{e_{i}}}$ and $\kappa_{i} \in \mathbb{Z}_{\geq 0}, \underline{\alpha}_{W_{i}}\left(\left|e_{i}\right|\right) \leq$ $W_{i}\left(e_{i}, \kappa_{i}\right) \leq \bar{\alpha}_{W_{i}}\left(\left|e_{i}\right|\right)$.

(ii) For any $\left(e_{i}, x, \kappa_{i}\right) \in \mathbb{R}^{n_{e_{i}}} \times \mathbb{R}^{n_{x}} \times \mathbb{Z}_{\geq 0}$, $W_{i}\left(\chi_{i}\left(e_{i}, \kappa_{i}\right), \kappa_{i}+1\right) \leq \rho_{i} W_{i}\left(e_{i}, \kappa_{i}\right)$.

(iii) For almost all $e_{i} \in \mathbb{R}^{n_{e_{i}}}$, all $\kappa_{i} \in \mathbb{Z}_{\geq 0}$ and $(x, w) \in \mathbb{R}^{n_{x}} \times \mathbb{R}^{n_{w}},\left\langle\frac{\partial W_{i}\left(e_{i}, \kappa_{i}\right)}{\partial e_{i}}, g_{i}(x, e, w)\right\rangle \leq$ $L_{W_{i}} W_{i}\left(e_{i}, \kappa_{i}\right)+\widetilde{H}_{i}(x, e, w)$ with $g_{i}$ coming from (11).

Items (i) and (ii) are exclusively related to the scheduling protocol implemented on network $\mathcal{N}_{i}$. Indeed, these items state that the protocol is uniformly globally asymptotically stable, see Definition 1 in [25]. These conditions are always satisfied for the sampled-data case, RR and TOD protocols for which expressions of $W_{i}$ are available, see [20]. Then given $W_{i}$, item (iii) of Assumption 1 essentially requires that $W_{i}$ exponentially grows on flows. Such a property is natural, as the $e_{i}$-system is typically unstable between two transmission instants. Item (iii) of Assumption 1 is always feasible when $W_{i}$ is globally Lipschitz in $e_{i}$ uniformly in $\kappa_{i}$, and $g_{i}$ satisfies a linear growth condition for instance, see Remark 11 in [20].

We assume that controller (2) has been designed to robustly stabilize system (1) in the following sense.

Assumption 2: There exist a locally Lipschitz function $V$ : $\mathbb{R}^{n_{x}} \rightarrow \mathbb{R}_{>0}, \underline{\alpha}_{V}, \bar{\alpha}_{V}, \alpha_{W} \in \mathcal{K}_{\infty}$, locally Lipschitz functions $\delta_{i}: \mathbb{R}^{n_{e_{i}}} \rightarrow \mathbb{R}_{\geq 0}$ satisfying $\delta_{i}(0)=0$, continuous functions $\widetilde{\alpha}_{V}: \mathbb{R}^{n_{x}} \times \mathbb{R}^{n_{e}} \times \mathbb{R}^{n_{w}} \rightarrow \mathbb{R}$ and $J_{i}: \mathbb{R}^{n_{x}} \times \mathbb{R}^{n_{e}} \times \mathbb{R}^{n_{w}} \rightarrow \mathbb{R}_{\geq 0}$, $\gamma_{i}>0, L_{\delta_{i}} \in \mathbb{R}, i \in \bar{N}$, such that the following hold.

(i) For all $x \in \mathbb{R}^{n_{x}}, \underline{\alpha}_{V}(|x|) \leq V(x) \leq \bar{\alpha}_{V}(|x|)$.

(ii) For almost all $x \in \mathbb{R}^{n_{x}}$ and all $(e, w) \in$ $\mathbb{R}^{n_{e}} \times \mathbb{R}^{n_{w}},\langle\nabla V(x), f(x, e, w)\rangle \leq-\widetilde{\alpha}_{V}(x, e, w)+$

${ }^{4}$ See the definition of $\|w\|_{\mathcal{L}_{p}}$ in [4]. 
$\sum_{i=1}^{N}\left(\gamma_{i}^{2} W_{i}^{2}\left(e_{i}, \kappa_{i}\right)-\widetilde{H}_{i}^{2}(x, e, w)-J_{i}(x, e, w)-\delta_{i}\left(v_{i}\right)\right)$, where $W_{i}$ and $\widetilde{H}_{i}$ come from Assumption 1 .

(iii) For almost all $x \in \mathbb{R}^{n_{x}}$ and all $(e, w) \in \mathbb{R}^{n_{e}} \times \mathbb{R}^{n_{w}}$, $\left\langle\nabla \delta_{i}\left(v_{i}\right), f_{v_{i}}(x, e, w)\right\rangle \leq L_{\delta_{i}} \delta_{i}\left(v_{i}\right)+\widetilde{H}_{i}^{2}(x, e, w)+$ $J_{i}(x, e, w)$ with $f_{v_{i}}$ coming from (11).

Assumption 2 states properties of the closed-loop system (1)-(2) and it neither requires any knowledge on the network, nor it implies the stability of (9). Indeed, variable $e$ is here understood as a generic perturbation affecting $(y, u)$. To verify whether Assumption 2 holds, we simply have to take the Lyapunov function $V$ used to ensure the stability of (1)-(2) in the absence of network, and study whether the required conditions are verified.

Function $\widetilde{\alpha}_{V}$ in Assumption 2 will be taken in the following as $\widetilde{\alpha}_{V}(x, e, w)=\alpha_{V}(|x|)+\alpha_{W}(|e|)-\sum_{i=1}^{N} \varrho_{V, i}(|w|)$ for some $\alpha_{V}, \alpha_{W}, \varrho_{V, i} \in \mathcal{K}_{\infty}, i \in \bar{N}$, when investigating ISS, and as $\widetilde{\alpha}_{V}(x, e, w)=-\mu\left(\theta^{p}|w|^{p}-|z|^{p}\right)$ with $\mu>0, \theta \geq 0$, when studying $\mathcal{L}_{p}$-stability. Item (ii) of Assumption 2 means that either the origin of (1)-(2) is ISS with respect to input $(e, w)$ or (1)-(2) is $\mathcal{L}_{p}$-stable from $w$ to $z$. That type of conditions are natural as we approach the problem by emulation, that is, the original closed-loop system needs to satisfy some robustness properties to cope with the errors induced by the network, as does any nonlinear controller, which is implemented in practice. But again, this does not mean that (9) satisfies desired stability properties because the $e$-system is typically unstable. Similar assumptions as item (ii) of Assumption 2 are often made in the literature on NCS, see, e.g., [4], [10], [28], where examples of systems satisfying these conditions are provided. The functions $\delta_{i}$ in Assumption 2 will be used to define the local event triggering condition. Item (iii) is an exponential growth condition of $\delta_{i}$ on flows, where the function $J_{i}$ is used to collect the redundant terms when we bound the norm of the derivative of $\delta_{i}\left(v_{i}\right)$ with $L_{\delta_{i}} \delta_{i}\left(v_{i}\right)+\widetilde{H}_{i}^{2}(x, e, w)$.

We show in Section V how to satisfy Assumptions 1 and 2 for a class of globally Lipschitz systems. A nonlinear example, which is not globally Lipschitz and satisfies all the required conditions, is provided in Section VI.

Remark 1: Assumptions 1-2 may be verified by systems subject to model uncertainties. Indeed, these Lyapunov-like conditions do not necessarily require a precise model of the plant to checked, as will be illustrated in Section VI.

Remark 2: Assumptions 1-2 impose conditions on the class of systems to which the results apply. It is possible to relax these assumptions to only hold in a given compact set. In this case, the forthcoming results can be adapted to derive local stability properties, at the price of more technicalities, which we do not present in order not to blur the main message of the paper.

\section{B. Local triggering generators}

We exploit Assumptions 1-2 to design the triggering generators and $T_{i}, i \in \bar{N}$. We define $\Upsilon_{i}$ in (5) as, for $v_{i}, e_{i} \in \mathbb{R}^{n_{e_{i}}}$ and $\kappa_{i} \in \mathbb{Z}_{\geq 0}$,

$$
\Upsilon_{i}\left(e_{i}, v_{i}, \kappa_{i}\right)=\gamma_{i} W_{i}^{2}\left(e_{i}, \kappa_{i}\right)-\lambda_{i} \bar{\rho}_{i} \delta_{i}\left(v_{i}\right),
$$

where $\bar{\rho}_{i}:=\max \left\{\rho_{i}, \frac{\gamma_{i} \lambda_{i}}{1-\lambda_{i} L_{\delta_{i}}}\right\} \geq 0$ with $\rho_{i}$ and $W_{i}$ coming from Assumption $1, L_{\delta_{i}} \in \mathbb{R}, \gamma_{i}>0, \delta_{i}$ coming from Assumption 2, and $\lambda_{i} \geq 0$ is a design parameter. The triggering condition (13) is similar to those proposed in [3], [4], [10], [11] for CETC in different contexts. Note that $\bar{\rho}_{i}$ in (13) depends on $\rho_{i}$ and thus on the scheduling protocol. The mapping $\Upsilon_{i}$ only depends on the local variables $e_{i}, v_{i}$ and $\kappa_{i}$, and not the whole state $q$, which is essential for the envisioned setup and for the decentralized implementation of the triggering rule.

We select $\lambda_{i}$ in (13) such that $\lambda_{i}<\lambda_{i}^{*}$, where $\lambda_{i}^{*}$ is defined as

$$
\lambda_{i}^{*}:= \begin{cases}1 & \text { when } L_{\delta_{i}} \leq-\gamma_{i} \\ \min \left\{1, \frac{1}{L_{\delta_{i}}+\gamma_{i}}\right\} & \text { when } L_{\delta_{i}}>-\gamma_{i} .\end{cases}
$$

Given $\lambda_{i} \in\left[0, \lambda_{i}^{*}\right)$, we select $T_{i}$ defined after (9) such that $T_{i}<T_{\mathrm{MASP}, i}\left(\lambda_{i}\right)$, where $T_{\mathrm{MASP}, i}\left(\lambda_{i}\right)$ is the maximum allowable sampling period (MASP) of network $\mathcal{N}_{i}$ and is defined as

$$
T_{\mathrm{MASP}, i}\left(\lambda_{i}\right):= \begin{cases}\frac{1}{L_{W_{i}} r_{i}} \arctan \left(\vartheta_{i}\right), & \text { when } \gamma_{i}>L_{W_{i}} \\ \frac{1}{L_{W_{i}}} \frac{1}{1+\bar{\rho}_{i}}, & \text { when } \gamma_{i}=L_{W_{i}} \\ \frac{1}{L_{W_{i}} r_{i}} \operatorname{arctanh}\left(\vartheta_{i}\right), & \text { when } \gamma_{i}<L_{W_{i}}\end{cases}
$$

where $\bar{\rho}_{i}$ is defined above, $r_{i}:=\sqrt{\left|\left(\frac{\gamma_{i}}{L_{W_{i}}}\right)^{2}-1\right|}, \vartheta_{i}:=$ $\frac{r_{i}\left(1-\bar{\rho}_{i}\right)}{2 \frac{\bar{\rho}_{i}}{1+\bar{\rho}_{i}}\left(\frac{\gamma_{i}}{L_{W_{i}}}-1\right)+1+\bar{\rho}_{i}}, L_{W_{i}} \geq 0$ and $\gamma_{i}>0$ come respectively from Assumptions 1 and 2.

The bound in (15) depends on the triggering parameter $\lambda_{i}$. More precisely, the bound is decreasing in $\lambda_{i}$. In other words, the larger the $\lambda_{i}$, the smaller $T_{\mathrm{MASP}, i}\left(\lambda_{i}\right)$ and vice versa.

Remark 3: The local event-triggering condition in (13) ensures that the protocol equation (6) is input-to-state stable (ISS) with respect to $v_{i}$, see Definition 5.3 in [29]. In particular, in view of the definition of $\Upsilon_{i}$ in (13) and item (ii) of Assumption 1, a transmission is triggered when $W_{i}\left(e_{i}, \kappa_{i}\right) \geq$ $\sqrt{\lambda_{i} \bar{\rho}_{i} \delta_{i}\left(v_{i}\right) / \gamma_{i}}$, which ensures that $W_{i}\left(\chi_{i}\left(e_{i}, \kappa_{i}\right), \kappa_{i}+1\right) \leq$ $\rho_{i} W_{i}\left(e_{i}, \kappa_{i}\right)$, where $\rho_{i} \in[0,1)$, for any $v_{i}, e_{i} \in \mathbb{R}^{n_{e_{i}}}$. Although the actual protocol equation (6), which is implemented, is ISS, the scheduling rule itself, which is modeled by $\chi_{i}$ and decides which nodes gets access to the network is uniformly globally asymptotically stable (UGAS) in view of items (i) and (ii) of Assumption 1, see the definition of UGAS protocols in Remark 7 of [20].

Remark 4: When $\lambda_{i}=0, i \in \bar{N}$, the triggering function $\Upsilon_{i}$ is always non-negative. Consequently, transmissions occur at every sampling instant according to (5). We then recover the time-triggered results of [28], in particular the bound on the maximal allowable transmission interval (MATI) is the same 
when a single network is used and there are no disturbances, i.e. $w=0$, as well as those in [27] when the network consists of a single node.

\section{Input-to-state stability}

We are ready to state the next result about the input-to-state stability of system (9).

Theorem 1: Consider system (9) and suppose the following hold.

1) Assumption 1 holds with $\widetilde{H}_{i}(x, e, w)=H_{i}(x, e)+$ $\varrho_{W_{i}}(|w|)$ for some continuous functions $H_{i}: \mathbb{R}^{n_{x}} \times$ $\mathbb{R}^{n_{e}} \rightarrow \mathbb{R}_{\geq 0}$ and $\varrho_{W_{i}} \in \mathcal{K}_{\infty}, i \in \bar{N}$.

2) Assumption 2 holds with $\widetilde{\alpha}_{V}(x, e, w)=\alpha_{V}(|x|)+$ $\alpha_{W}(|e|)-\sum_{i=1}^{N} \varrho_{V, i}(|w|)$ for some $\alpha_{V}, \alpha_{W}, \varrho_{V, i} \in \mathcal{K}_{\infty}$, $i \in \bar{N}$

3) For each $i \in \bar{N}$, let $\lambda_{i} \in\left[0, \lambda_{i}^{*}\right)$ and $T_{i} \in$ $\left[\varepsilon_{i}, T_{\mathrm{MASP}, i}\left(\lambda_{i}\right)\right)$, where $\lambda_{i}^{*}$ and $T_{\mathrm{MASP}, i}\left(\lambda_{i}\right)$ are defined in (14) and (15), respectively.

Then, the set $\mathcal{A}:=\left\{q \in C \cup D: x=0, e=0, \kappa_{i} \in \mathbb{Z}_{>0}, \tau_{i} \in\right.$ $\left.\left[0, T_{i}\right], i \in \bar{N}\right\}$ is ISS for system (9).

Theorem 1, whose proof is given in Appendix A, shows that set $\mathcal{A}$ is ISS for system (9). This implies that (i) $x$ and $e$ globally converge to a neighborhood of the origin, whose "size" depends on the norm of the disturbance $w$; (ii) the set $\mathcal{A}$ is uniformly globally asymptotically stable [22, Definition 3.6] when $w=0$.

Remark 5: Theorem 1 relies on small-gain techniques. The general idea is that the $x$-system is assumed to satisfy an ISS property with respect to $\left(w, W_{1}\left(e_{1}, \kappa_{1}\right), \ldots, W_{N}\left(e_{N}, \kappa_{N}\right)\right)$ on flows according to items (i)-(ii) of Assumption 2, and remains constant at jumps. On the other hand, Assumption 1 leads to an ISS property of the $e$-system with respect to $(x, w)$ as well, as shown in Proposition 6 in [20], thanks to the definition of the event generators. Then, by carefully selecting the triggering conditions and $T_{i}$, the small gain condition apply and the desired result is obtained. While the connection with smallgain techniques is easier to see in the case where the controller is a state-feedback law and there is only one network as in [24], the fact that output-feedback control is addressed and the decentralized scenario we investigate prevent us to directly apply existing hybrid small-gain results. That is the reason why we propose a completely novel hybrid Lyapunov construction in the proof of Theorem 1.

Tailored results can be derived from Theorem 1 either under stronger conditions or for more specific implementation setups. Thus, an exponential-ISS property is obtained by strengthening the conditions of Theorem 1 as stated next, whose proof follows directly from the proof of Theorem 1 and is therefore omitted.

Corollary 1: Consider system (9). Suppose that items 1) and 2) of Theorem 1 are satisfied and there exist $\underline{a}_{W_{i}}, \bar{a}_{W_{i}}, \underline{a}_{V}, \bar{a}_{V}, a_{V}, a_{W}>0, i \in \bar{N}$, such that Assumptions 1 and 2 , respectively, hold with $\underline{\alpha}_{W_{i}}(s)=\underline{a}_{W_{i}} s, \bar{\alpha}_{W_{i}}(s)=$ $\bar{a}_{W_{i}} s, \underline{\alpha}_{V}(s)=\underline{a}_{V} s^{2}, \bar{\alpha}_{V}(s)=\bar{a}_{V} s^{2}, \alpha_{V}(s)=a_{V} s^{2}$ and $\alpha_{W}(s)=a_{W} s^{2}$ for $s \geq 0$. Then, the set $\mathcal{A}$ defined in Theorem 1 is exponentially-ISS with a linear gain.
When a single network is used and the state of plant $x_{p}$ is available for control, i.e. $y=x_{p}$ in (1), we can relax Assumption 2 and modify the triggering condition. Since we consider only one network here, only one triggering generator is needed. We therefore use the notation $\Upsilon$ to define the triggering condition, and $\chi$ to denote the scheduling rule. As we need to specify the expressions of $\widetilde{H}_{i}, J_{i}$ in Assumptions 1 and 2 for this special case, we rewrite those conditions here as follows.

Assumption 3: There exist locally Lipschitz functions $V$ : $\mathbb{R}^{n_{x}} \rightarrow \mathbb{R}_{\geq 0}$ and $W: \mathbb{R}^{n_{e}} \times \mathbb{Z}_{\geq 0} \rightarrow \mathbb{R}_{\geq 0}$ with $V$ and $W$ positive definite, $\varrho_{W} \in \mathcal{K}_{\infty}$, continuous function $\widetilde{\alpha}_{V}: \mathbb{R}^{n_{x}} \times$ $\mathbb{R}^{n_{e}} \times \mathbb{R}^{n_{w}} \rightarrow \mathbb{R}_{\geq 0}, \rho \in[0,1), a_{V}, \gamma>0$, and $L_{W}, L_{V} \geq 0$ such that the following hold.

(i) For any $(e, x, \kappa) \in \mathbb{R}^{n_{e}} \times \mathbb{R}^{n_{x}} \times \mathbb{Z}_{\geq 0}, W(\chi(e, \kappa), \kappa+$ $1) \leq \rho W(e, \kappa)$.

(ii) For almost all $e \in \mathbb{R}^{n_{e}}$, all $\kappa \in \mathbb{Z}_{>0}$ and $(x, w) \in$ $\mathbb{R}^{n_{x}} \times \mathbb{R}^{n_{w}},\left\langle\frac{\partial W(e, \kappa)}{\partial e}, g(x, e, w)\right\rangle \leq L_{W} W(e, \kappa)+$ $L_{V} \sqrt{V(x)}+\varrho_{W}(|w|)$.

(iii) For almost all $x \in \mathbb{R}^{n_{x}}$ and all $(e, w) \in \mathbb{R}^{n_{e}} \times$ $\mathbb{R}^{n_{w}},\langle\nabla V(x), f(x, e, w)\rangle \leq-a_{V} V(x)-\widetilde{\alpha}_{V}(x, e, w)+$ $\gamma^{2} W^{2}(e, \kappa)$.

We define the single triggering condition $\Upsilon$, as for $(x, e) \in$ $\mathbb{R}^{n_{x}} \times \mathbb{R}^{n_{e}}$ and $\kappa \in \mathbb{Z}_{\geq 0}$,

$$
\Upsilon(e, x, \kappa)=\gamma W^{2}(e, \kappa)-\lambda \bar{\rho} V(x)
$$

where $\bar{\rho}:=\max \left\{\rho, \frac{\gamma \lambda}{a_{V}}\right\}$. We select $\lambda$ such that $\lambda<\lambda^{*}$ with

$$
\lambda^{*}:=\min \left\{1, \frac{a_{V}}{\gamma}\right\} \text {. }
$$

For each $\lambda \in\left[0, \lambda^{*}\right)$, the $\operatorname{MASP} T_{\mathrm{MASP}}(\lambda)$ is defined as

$$
T_{\mathrm{MASP}}(\lambda):= \begin{cases}\frac{1}{L_{W} r} \arctan (\vartheta), & \text { when } \gamma L_{V}>L_{W}, \\ \frac{1}{L_{W}} \frac{1-\bar{\rho}}{1+\bar{\rho}}, & \text { when } \gamma L_{V}=L_{W}, \\ \frac{1}{L_{W} r} \operatorname{arctanh}(\vartheta), & \text { when } \gamma L_{V}<L_{W},\end{cases}
$$

where $\bar{\rho}$ is defined below $(16), r:=\sqrt{\left|\left(\frac{\gamma L_{V}}{L_{W} \sqrt{a_{V}}}\right)^{2}-1\right|}$, $\vartheta:=\frac{r(1-\bar{\rho})}{2 \frac{\bar{\rho}}{1+\bar{\rho}}\left(\frac{\gamma}{L_{W}} \frac{L_{V}^{2}+a_{V}}{2 a_{V}}-1\right)+1+\bar{\rho}}, L_{W}, L_{V} \geq 0$, and $a_{V}, \gamma>0$ come from Assumption 3. The MASP in (18) is different to (15) since extra parameters $L_{V} \geq 0$ and $a_{V}>0$ are introduced in Assumption 3 and it is consistent with (15) when $L_{V}^{2} / a_{V}=1$.

We can state the next theorem.

Theorem 2: Consider system (9). Suppose that Assumption 3 holds with $\widetilde{\alpha}_{V}(x, e, w)=\alpha_{W}(|e|)-\varrho_{V}(|w|)$ for some $\alpha_{W}, \varrho_{V} \in \mathcal{K}_{\infty}$ from Assumption 3. Let $\lambda \in\left[0, \lambda^{*}\right)$ and $T<T_{\mathrm{MASP}}(\lambda)$, where $\lambda^{*}$ and $T_{\mathrm{MASP}}(\lambda)$ are defined in (17) and (18), respectively. Then, set $\mathcal{A}$ defined in Theorem 1 is ISS. 
Theorem 2 extends the main result of [23] to scheduling and allows considering exogenous disturbances acting on the plant and [24] to scheduling. Note that the conditions in [24] which are parallel to Assumption 3 are slightly different, where $\rho=0$ and $\widetilde{H}_{i}, J_{i}$ are not specified as in Assumption 3 .

\section{D. $\mathcal{L}_{p}$-stability}

We now consider the input-output stability of system (9) from $w$ to the output $z=\eta(x, w)$.

Theorem 3: Consider system (9) and suppose the following hold.

1) Assumptions 1 and 2 are verified with $\widetilde{\alpha}_{V}(x, e, w)=$ $-\mu\left(\theta^{p}|w|^{p}-|\eta(x, w)|^{p}\right)$ for some $\mu, \theta>0$.

2) For each $i \in \bar{N}$, let $\lambda_{i} \in\left[0, \lambda_{i}^{*}\right)$ and $T_{i}<T_{\mathrm{MASP}, i}\left(\lambda_{i}\right)$, where $\lambda_{i}^{*}$ and $T_{\mathrm{MASP}, i}\left(\lambda_{i}\right)$ are defined in (14) and (15), respectively.

Then system (9) is $\mathcal{L}_{p}$-stable from $w$ and to $z$ with respect to the set $\mathcal{A}$ defined in Theorem 1 with gain less than or equal to $\theta$.

Like in Section IV-C, we derive a tailored result for the case where a single network is used and $y=x_{p}$. Its proof follows from the ones to Theorems 2 and 3, and is therefore again omitted.

Theorem 4: Consider system (9). Suppose that Assumption 3 holds with $\widetilde{\alpha}_{V}(x, e, w)=-\mu\left(\theta^{p}|w|^{p}-|\eta(x, w)|^{p}\right)$ for some $\mu, \theta>0$. Let $\lambda \in\left[0, \lambda^{*}\right)$ and $T<T_{\mathrm{MASP}}(\lambda)$, where $\lambda^{*}$ and $T_{\text {MASP }}(\lambda)$ are defined in (17) and (18), respectively. Then, system (9) is $\mathcal{L}_{p}$-stable from $w$ and to $z$ with respect to the set $\mathcal{A}$ defined in Theorem 1 with gain less than or equal to $\theta$.

\section{CASE STUdY}

\section{A. Setup and hybrid model}

We consider nonlinear systems of the form

$$
\begin{aligned}
\dot{x}_{p} & =A_{p} x_{p}+B_{p} u+D_{p} \psi\left(x_{p}\right)+E_{p} w \\
y & =C_{p} x_{p},
\end{aligned}
$$

where $x_{p} \in \mathbb{R}^{n_{p}}$ is the state, $u \in \mathbb{R}^{n_{u}}$ is the control input, $w \in \mathbb{R}^{n_{w}}$ is the external disturbance, $y \in \mathbb{R}^{n_{y}}$ is the measured output, $A_{p}, B_{p}, C_{p}, D_{p}$ and $E_{p}$ are matrices of appropriate dimensions, $\left(A_{p}, B_{p}\right)$ and $\left(A_{p}, C_{p}\right)$ are assumed to be stabilizable and detectable, respectively, and $\psi: \mathbb{R}^{n_{p}} \rightarrow \mathbb{R}^{n_{r}}$, satisfies $\psi(0)=0$ and $\left|\psi\left(x_{1}\right)-\psi\left(x_{2}\right)\right| \leq L\left|x_{1}-x_{2}\right|$ for all $x_{1}, x_{2} \in \mathbb{R}^{n_{p}}$ with constant $L>0$, where $n_{r} \in \mathbb{Z}_{>} 0$.

We focus on observer-based controllers of the form

$$
\begin{aligned}
\dot{x}_{c} & =A_{p} x_{c}+B_{p} u+D_{p} \psi\left(x_{c}\right)-M\left(C_{p} x_{c}-y\right) \\
u & =K x_{c},
\end{aligned}
$$

where $x_{c} \in \mathbb{R}^{n_{p}}$ is the state estimate, $M$ and $K$ are matrices of appropriate dimensions such that $\mathcal{A}_{1}:=$ $\left[\begin{array}{ll}A_{p} & B_{p} K \\ M C_{p} & A_{p}+B_{p} K-M C_{p}\end{array}\right]$ is Hurwitz, which is always possible since $\left(A_{p}, B_{p}\right)$ and $\left(A_{p}, C_{p}\right)$ are stabilizable and detectable, respectively.

We consider the scenario where the plant and the controller communicate via $N$ independently operating networks, as described in Section III. Zero-order-hold devices are used so that $\hat{f}_{v}=0$ as defined after (4). Each network is scheduled by an arbitrary uniformly globally exponentially stable (UGES) protocol, whenever the local triggering rule is satisfied. Hence, items (i) and (ii) of Assumption 1 hold

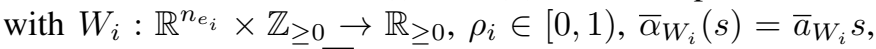
$\underline{\alpha}_{W_{i}}(s)=\underline{a}_{W_{i}} s, i \in \bar{N}$, for some $\bar{a}_{W_{i}} \geq \underline{a}_{W_{i}}>0$ and all $s \geq 0$, which depend on the considered protocol. We further assume that there exists $\varpi_{i} \geq 0$ such that $\left|\frac{\partial W_{i}\left(e_{i}, \kappa_{i}\right)}{\partial e_{i}}\right| \leq \varpi_{i}$ for almost all $e_{i} \in \mathbb{R}^{n_{e_{i}}}$ and $\kappa_{i} \in \mathbb{Z}_{\geq 0}$, which is the case for the sampled-data case and RR and TOD protocols according to Section V in [20].

Let $x=\left(x_{p}, x_{c}\right) \in \mathbb{R}^{n_{x}}, v=(y, u) \in \mathbb{R}^{n_{e}}, e=(\hat{v}-v)$, $n_{x}=n_{p}+n_{c}$ and $n_{e}=n_{y}+n_{u}$. We write $v:=\bar{C} x, \bar{C}:=$

$$
\begin{aligned}
& {\left[\begin{array}{cc}
C_{p} & 0 \\
0 & K
\end{array}\right] \in \mathbb{R}^{n_{e} \times n_{x}} \text { and }} \\
& \qquad v_{i}:=\bar{C}_{i} x \in \mathbb{R}^{n_{e_{i}}}, \quad i \in \bar{N},
\end{aligned}
$$

be the $i$-th element of $v$ associated with network $\mathcal{N}_{i}$ with $\bar{C}_{i} \in \mathbb{R}^{n_{e_{i}} \times n_{x}}$. Define $e_{y}:=\hat{y}-y=C_{y} e, e_{u}:=\hat{u}-u=C_{u} e$, with appropriate matrices $C_{y} \in \mathbb{R}^{n_{y} \times n_{e}}$ and $C_{u} \in \mathbb{R}^{n_{u} \times n_{e}}$.

In this case, the hybrid model (9) is given by,

$$
\begin{aligned}
& \dot{q}=\left(\begin{array}{c}
\mathcal{A}_{1} x+\mathcal{B}_{1} e+\mathcal{D}_{1} \bar{\psi}(x)+\mathcal{E}_{1} w \\
\mathcal{A}_{2} x+\mathcal{B}_{2} e+\mathcal{D}_{2} \bar{\psi}(x)+\mathcal{E}_{2} w \\
\mathbf{0}_{N} \\
\mathbf{1}_{N}
\end{array}\right) \quad q \in C \\
& q^{+} \in G(q) \quad q \in D,
\end{aligned}
$$

where $q=(x, e, \kappa, \tau), \quad \mathcal{B}_{1}:=\left[\begin{array}{c}B_{p} C_{u} \\ M C_{y}\end{array}\right], \quad \mathcal{D}_{1}:=$ $\left[\begin{array}{cc}D_{p} & 0 \\ 0 & D_{p}\end{array}\right], \mathcal{E}_{1}:=\left[\begin{array}{c}E_{p} \\ 0\end{array}\right], \bar{\psi}(x):=\left(\psi\left(x_{p}\right), \psi\left(x_{c}\right)\right)$, $\mathcal{A}_{2}:=-\left[\begin{array}{c}\bar{C}_{1} \mathcal{A}_{1} \\ \vdots \\ \bar{C}_{N} \mathcal{A}_{1}\end{array}\right], \mathcal{B}_{2}:=-\left[\begin{array}{c}\bar{C}_{1} \mathcal{B}_{1} \\ \vdots \\ \bar{C}_{N} \mathcal{B}_{1}\end{array}\right], \mathcal{D}_{2}:=$ $-\left[\begin{array}{c}\bar{C}_{1} \mathcal{D}_{1} \\ \vdots \\ \bar{C}_{N} \mathcal{D}_{1}\end{array}\right], \mathcal{E}_{2}:=-\left[\begin{array}{c}\bar{C}_{1} \mathcal{E}_{1} \\ \vdots \\ \bar{C}_{N} \mathcal{E}_{1}\end{array}\right]$. The flow and jump sets $C$ and $D$ are defined after (9), and the jump map $G$ is given in (12).

\section{B. Input-to-state stability}

Before stating the main results of this section, we need to introduce some notation. For any $\pi=\left(\pi_{1}, \ldots, \pi_{N}\right) \in \mathbb{R}_{\geq 0}^{N}$, we define

$$
\Psi(\pi):=\operatorname{diag}\left\{\pi_{1} I_{n_{e_{1}}}, \ldots, \pi_{N} I_{n_{e_{N}}}\right\} \in \mathbb{R}^{n_{e} \times n_{e}} .
$$

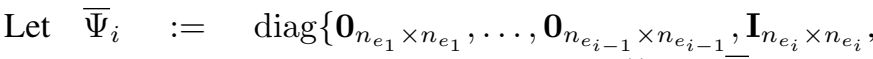
$\left.\mathbf{0}_{n_{e_{i+1}} \times n_{e_{i+1}}}, \ldots, \mathbf{0}_{n_{e_{N}} \times n_{e_{N}}}\right\} \in \mathbb{R}^{n_{e} \times n_{e}}, \quad \overline{\mathcal{B}}_{2, i}:=$ $\bar{C}_{i} \mathcal{B}_{1}\left(I_{n_{e}}-\bar{\Psi}_{i}\right) \in \mathbb{R}^{n_{e_{i}} \times n_{e}}, i \in \bar{N}, \overline{\mathcal{B}}_{2}:=\left[\begin{array}{c}\overline{\mathcal{B}}_{2,1} \\ \vdots \\ \overline{\mathcal{B}}_{2, N}\end{array}\right] \in$ $\mathbb{R}^{n_{e} \times n_{e}}$, where $\bar{C}_{i}$ comes from (21). 
TABLE I: Expression of functions and parameters in Section V.

\begin{tabular}{|c|c|c|}
\hline & Proposition 1/Lemma 1 & Proposition 2 \\
\hline$\Sigma_{11}:=$ & $\begin{array}{l}\mathcal{A}_{1}^{T} P+P \mathcal{A}_{1}+a_{V} I_{n_{x}}+\mathcal{D}_{2}^{T} \Psi^{T}(\sqrt{2} \varpi L) \Psi(\sqrt{2} \varpi L) \mathcal{D}_{2} \\
+2 L\left|\mathcal{D}_{1}\right| P+\mathcal{A}_{2}^{T}\left(\Psi^{T}(\varpi) \Psi(\varpi)+\Psi^{T}(\epsilon) \Psi(\epsilon)\right) \mathcal{A}_{2} \\
+\mathcal{D}_{2}^{T} \Psi^{T}(\sqrt{2 L} \varpi) \Psi(\sqrt{2 L} \varpi) \mathcal{A}_{2} \\
+\bar{C}^{T} \Psi^{T}\left(\epsilon \sqrt{2 L\left|\mathcal{D}_{1}\right|+1}\right) \Psi\left(\epsilon \sqrt{2 L\left|\mathcal{D}_{1}\right|+1}\right) \bar{C}\end{array}$ & $\begin{array}{l}\mathcal{A}_{1}^{T} P+P \mathcal{A}_{1}+\mathcal{D}_{2}^{T} \Psi^{T}(\varpi L) \Psi(\varpi L) \mathcal{D}_{2} \\
+2 L\left|\mathcal{D}_{1}\right| P+\mathcal{A}_{2}^{T}\left(\Psi^{T}(\varpi) \Psi(\varpi)+\Psi^{T}(\epsilon) \Psi(\epsilon)\right) \mathcal{A}_{2}^{T} \\
+\mathcal{D}_{2}^{T} \Psi^{T}(\sqrt{2 L} \varpi) \Psi(\sqrt{2 L} \varpi) \mathcal{A}_{2} \\
+\bar{C}^{T} \Psi^{T}\left(\epsilon \sqrt{2 L\left|\mathcal{D}_{1}\right|+1}\right) \Psi\left(\epsilon \sqrt{2 L\left|\mathcal{D}_{1}\right|+1}\right) \bar{C}+\mu C_{z}^{T} C_{z}\end{array}$ \\
\hline$\Sigma_{21}:=$ & $\mathcal{B}_{1}^{T} P+\overline{\mathcal{B}}_{2}^{T} \Psi^{T}(\varpi) \Psi(\varpi) \mathcal{A}_{2}+\mathcal{B}_{2}^{T} \Psi^{T}(\epsilon) \Psi(\epsilon) \bar{C}$ & $\mathcal{B}_{1}^{T} P+\overline{\mathcal{B}}_{2}^{T} \Psi^{T}(\varpi) \Psi(\varpi) \mathcal{A}_{2}+\mathcal{B}_{2}^{T} \Psi^{T}(\epsilon) \Psi(\epsilon) \bar{C}$ \\
\hline $\begin{array}{l}\Sigma_{22}:= \\
\Sigma_{31}:= \\
\Sigma_{23}:=\end{array}$ & $\begin{array}{l}-\left(\Psi(\nu)-a_{W} I_{n_{e}}\right)+\overline{\mathcal{B}}_{2}^{T} \Psi^{T}(\varpi) \Psi(\varpi) \overline{\mathcal{B}}_{2} \\
\mathcal{E}_{1}^{T} P+\mathcal{E}_{2}^{T} \Psi^{T}(\varpi) \Psi(\varpi) \mathcal{A}_{2}+\mathcal{E}_{2}^{T} \Psi^{T}(\epsilon) \Psi(\epsilon) \bar{C} \\
-\tilde{\theta} I\end{array}$ & $\begin{array}{l}-\Psi(\nu)+\overline{\mathcal{B}}_{2}^{T} \Psi^{T}(\varpi) \Psi(\varpi) \overline{\mathcal{B}}_{2} \\
\mathcal{E}_{1}^{T} P+\mathcal{E}_{2}^{T} \Psi^{T}(\varpi) \Psi(\varpi) \mathcal{A}_{2}+\mathcal{E}_{2}^{T} \Psi^{T}(\epsilon) \Psi(\epsilon) \bar{C}+\mu D_{z}^{T} C_{z}\end{array}$ \\
\hline $\begin{array}{l}\Sigma_{33}:= \\
\underline{\alpha}_{W_{i}}(s):=\end{array}$ & $\begin{array}{l}-\tilde{\theta} I_{n_{w}}+\mathcal{E}_{2}^{T} \Psi^{T}(\varpi) \Psi(\varpi) \mathcal{E}_{2}^{T} \\
\underline{a}_{W_{i}} s\end{array}$ & $\begin{array}{l}-\mu \theta^{2} I_{n_{w}}+\mu D_{z}^{T} D_{z}+\mathcal{E}_{2}^{T} \Psi^{T}(\varpi) \Psi(\varpi) \mathcal{E}_{2} \\
\underline{a}_{W_{j}} s\end{array}$ \\
\hline$\overline{\bar{\alpha}}_{W_{i}}(s):=$ & $\bar{a}_{W_{i}} s$ & $\overline{\bar{a}}_{W_{i}} s$ \\
\hline$L_{W_{i}}:=$ & $\varpi_{i} / \underline{a}_{W_{i}}\left|\bar{C}_{i} \mathcal{B}_{1} \bar{\Psi}_{i}\right|$ & $\varpi_{i} / \underline{a}_{W_{i}}\left|\bar{C}_{i} \mathcal{B}_{1} \bar{\Psi}_{i}\right|$ \\
\hline$H_{i}(x, e):=$ & $\varpi_{i}\left(\left|\bar{C}_{i} \mathcal{A}_{1} x+\overline{\mathcal{B}}_{2, i} e\right|+L\left|\bar{C}_{i} \mathcal{D}_{1}\right||x|\right)$ & $\varpi_{i}\left(\left|\bar{C}_{i} \mathcal{A}_{1} x+\overline{\mathcal{B}}_{2, i} e+L\right| \bar{C}_{i} \mathcal{D}_{1}|| x \mid\right)$ \\
\hline$\varrho_{W_{i}}(w):=$ & $\varpi_{i}\left|\bar{C}_{i} \mathcal{E}_{1}\right||w|$ & $\varpi_{i}\left|\bar{C}_{i} \mathcal{E}_{1} w\right|$ \\
\hline$V(x):=$ & $x^{T} P x$ & $x^{T} P x$ \\
\hline$\delta_{i}\left(v_{i}\right):=$ & $\epsilon_{i}^{2}\left|v_{i}\right|^{2}$ & $\delta_{i}\left(v_{i}\right)=\epsilon_{i}^{2}\left|v_{i}\right|^{2}$ \\
\hline$\underline{\alpha}_{V}(s):=$ & $\lambda_{\min }(P) s^{2}$ & $\lambda_{\min }(P) s^{2}$ \\
\hline$\overline{\bar{\alpha}}_{V}(s):=$ & $\lambda_{\max }(P) s^{2} \quad N$ & $\lambda_{\max }(P) s^{2}$ \\
\hline$\widetilde{\alpha}_{V}(x, e, w):=$ & $a_{V}|x|^{2}+a_{W}|e|^{2}-\sum_{i=1}^{N} \frac{1}{N} \tilde{\theta}|w|^{2}$ & $\mu\left(\left|C_{z} x+D_{z} w\right|^{2}-\theta^{2}|w|^{2}\right)$ \\
\hline$\gamma_{i}:=$ & $\sqrt{\frac{\nu_{i}-a_{W}-\varpi_{i}^{2}\left|\overline{\mathcal{B}}_{2, i}\right|^{2}}{\underline{a}_{W_{i}}^{2}}}$ & $\sqrt{\frac{\nu_{i}-\varpi_{i}^{2}\left|\overline{\mathcal{B}}_{2, i}\right|^{2}}{\underline{a}_{W_{i}}^{2}}}$ \\
\hline$J_{i}(x, e, w):=$ & $\begin{array}{l}\epsilon_{i}^{2}\left(\left|\mathcal{A}_{1}^{T} \bar{C}_{i}^{T} \bar{C}_{i} \mathcal{A}_{1}\right|+2 L\left|\bar{C}_{i}^{T} \bar{C}_{i} \mathcal{D}_{1}\right|\right)|x|^{2} \\
+2 \epsilon_{i}^{2}\left|x^{T} \bar{C}_{i}^{T} \bar{C}_{i}\left(\mathcal{B}_{1} e+\mathcal{E}_{1} w\right)\right|\end{array}$ & $\begin{array}{l}\epsilon_{i}^{2}\left(\left|\mathcal{A}_{1}^{T} \bar{C}_{i}^{T} \bar{C}_{i} \mathcal{A}_{1}\right|+2 L\left|\bar{C}_{i}^{T} \bar{C}_{i} \mathcal{D}_{1}\right|\right)|x|^{2} \\
+2 \epsilon_{i}^{2}\left|x^{T} \bar{C}_{i}^{T} \bar{C}_{i}\left(\mathcal{B}_{1} e+\mathcal{E}_{1} w\right)\right|\end{array}$ \\
\hline$L_{\delta_{i}}:=$ & 1 & 1 \\
\hline
\end{tabular}

The next proposition states that all conditions of Corollary 1 hold if the linear matrix inequality (LMI) in (24) is satisfied.

Proposition 1: If there exist a positive definite symmetric matrix $P, a_{V}, a_{W}, \tilde{\theta}, \underline{a}_{W_{i}}, \epsilon_{i}>0, \varpi_{i} \geq \underline{a}_{W_{i}}$ and $\nu_{i} \geq a_{W}+$ $\varpi_{i}^{2}\left|\overline{\mathcal{B}}_{2, i}\right|^{2}, i \in \bar{N}$, such that the following LMI holds

$$
\left[\begin{array}{ccc}
\Sigma_{11} & \star & \star \\
\Sigma_{21} & \Sigma_{22} & \star \\
\Sigma_{31} & \mathcal{E}_{2}^{T} \Psi^{T}(\varpi) \Psi(\varpi) \overline{\mathcal{B}}_{2} & \Sigma_{33}
\end{array}\right]<0,
$$

where $\Psi$ is defined in (23), $\varpi:=\left(\varpi_{1}, \ldots, \varpi_{N}\right), \epsilon:=$ $\left(\epsilon_{1}, \ldots, \epsilon_{N}\right), \Sigma_{11}, \Sigma_{21}, \Sigma_{22}, \Sigma_{31}$ and $\Sigma_{33}$ are given in Table I. Then, Assumptions 1-2 hold with the data given in Table I.

An immediate consequence of Proposition 1 is that the set $\mathcal{A}=\left\{q \in C \cup D: x=0, e=0, \kappa_{i} \in \mathbb{Z}_{\geq 0}, \tau_{i} \in\left[0, T_{i}\right], i \in\right.$ $\bar{N}\}$ is exponentially-ISS with a linear gain for system (22) according to Corollary 1 by suitably defining $\lambda_{i}$ and $T_{i}$, as Proposition 1 ensures the satisfaction of all the conditions of Corollary 1.

\section{C. $\mathcal{L}_{2}$-stability}

We now consider $\mathcal{L}_{2}$-stability for system (22) using Theorem 3 , with respect to the performance output $z:=C_{z} x+$ $D_{z} w$.

We can follow the proof of Proposition 1, provided in the Appendix A, to show that the conditions of Theorem 3 hold.

Proposition 2: If there exist a positive definite symmetric matrix $P, \mu, \theta, \epsilon_{i}, \underline{a}_{W_{i}}>0, \varpi_{i} \geq \underline{a}_{W_{i}}$ and $\nu_{i} \geq \varpi_{i}^{2}\left|\overline{\mathcal{B}}_{2, i}\right|^{2}$, $i \in \bar{N}$, such that (24) holds. Then, Assumptions 1-2 hold with the data given in Table I.
Based on Proposition 2, system (22) is $\mathcal{L}_{2}$-stable from $w$ to $z$ with respect to the set $\mathcal{A}$ with gain less than or equal to $\theta$ according to Theorem 3 , when $\lambda_{i}<\lambda_{i}^{*}$ and $T_{i}<T_{\mathrm{MASP}, i}\left(\lambda_{i}\right)$ with $\lambda_{i}^{*}$ in (14) and $T_{\mathrm{MASP}, i}\left(\lambda_{i}\right)$ in (15).

\section{Special cases}

When $\psi$ in (19) only depends on the output $y$, not the state $x_{p}$, and $D_{p}=B_{p}$, condition (24) slightly differs and can be shown to always hold as formalized next.

Lemma 1: Consider system (19) with $\psi(y)$ instead of $\psi\left(x_{p}\right)$, and $D_{p}=B_{p}$. Let $\mathcal{D}_{1}:=\left[\begin{array}{cc}B_{p} & 0 \\ 0 & 0\end{array}\right]$ and replace $\bar{\psi}(x)$ in (22) by $\bar{\psi}(y, e):=\left[\begin{array}{c}\psi(y)-\psi\left(y+e_{y}\right) \\ 0\end{array}\right]$. Then, there exist a positive definite symmetric matrix $P, a_{V}, a_{W}, \tilde{\theta}, \underline{a}_{W_{i}}, \epsilon_{i}>0$, $\varpi_{i} \geq \underline{a}_{W_{i}}$ and $\nu_{i} \geq a_{W}+L^{2}\left|\mathcal{D}_{1}\right|^{2}|P|^{2} / a_{V}+\varpi_{i}^{2}\left|\overline{\mathcal{B}}_{2, i}\right|^{2}+$ $\epsilon_{i}^{2} L^{2}\left|\bar{C}_{i} \mathcal{D}_{1}\right|^{2}+2 \varpi_{i}^{2}\left(L^{2}\left|\bar{C}_{i} \mathcal{D}_{1}\right|^{2}+L\left|\bar{C}_{i} \mathcal{D}_{1}\right|\left|\overline{\mathcal{B}}_{2, i}\right|\right), i \in \bar{N}$, such that (24) holds with $\Sigma_{21}, \Sigma_{31}$ and $\Sigma_{33}$ from Table $\mathrm{I}$ and $\Sigma_{11}:=\mathcal{A}_{1}^{T} P+P \mathcal{A}_{1}+\mathcal{A}_{2}^{T}\left(\Psi^{T}(\sqrt{2} \varpi) \Psi(\sqrt{2} \varpi)+\right.$ $\left.\Psi^{T}(\epsilon) \Psi(\epsilon)\right) \mathcal{A}_{2}+2 a_{V} I_{n_{x}}+\bar{C}^{T} \Psi^{T}(\epsilon) \Psi(\epsilon) \bar{C}, \quad \Sigma_{22} \quad:=$ $-\Psi(\nu)+\left(a_{W}+L^{2}\left|\mathcal{D}_{1}\right|^{2}|P|^{2} / a_{V}\right) I_{n_{e}}+\overline{\mathcal{B}}_{2}^{T} \Psi^{T}(\varpi) \Psi(\varpi) \overline{\mathcal{B}}_{2}+$ $\Psi^{T}\left(\epsilon L\left|\mathcal{D}_{2}\right|\right) \Psi\left(\epsilon L\left|\mathcal{D}_{2}\right|\right)+\Psi^{T}(k \varpi) \Psi^{T}(k \varpi) \quad$ with $k^{2}=2 L^{2}\left|\mathcal{D}_{2}\right|^{2}+2 L\left|\mathcal{D}_{2}\right| \overline{\mathcal{B}}_{2} \mid$. As a result, Assumptions 1-2 hold with the data from Table I except that $H_{i}(x, e) \quad:=\varpi_{i}\left(\left|\bar{C}_{i} \mathcal{A}_{1} x+\overline{\mathcal{B}}_{2, i} e\right|+L\left|\bar{C}_{i} \mathcal{D}_{2}\right||e|\right)$, $\gamma_{i}^{2}=\left(\nu_{i}-\left(a_{W}+L^{2}\left|\mathcal{D}_{1}\right|^{2}|P|^{2} / a_{V}+\varpi_{i}^{2}\left|\overline{\mathcal{B}}_{2, i}\right|^{2}+\right.\right.$ $\left.\left.\epsilon_{i}^{2} L^{2}\left|\bar{C}_{i} \mathcal{D}_{1}\right|^{2}+2 \varpi_{i}^{2}\left(L^{2}\left|\bar{C}_{i} \mathcal{D}_{1}\right|^{2}+L\left|\bar{C}_{i} \mathcal{D}_{1}\right|\left|\overline{\mathcal{B}}_{2, i}\right|\right)\right)\right) / \underline{a}_{W_{i}}^{2}$, $J_{i}(x, e, w) \quad:=\epsilon_{i}^{2}\left(\left(\left|\mathcal{A}_{1}^{T} \bar{C}_{i}^{T} \bar{C}_{i} \mathcal{A}_{1}\right|+\left|\bar{C}_{i}\right|^{2}\right)|x|^{2}+\right.$ $\left.L^{2}\left|\bar{C}_{i} \mathcal{D}_{1}\right|^{2}|e|^{2}\right)+2 \epsilon_{i}^{2}\left|x^{T} \bar{C}_{i}^{T} \bar{C}_{i}\left(\mathcal{B}_{1} e+\mathcal{E}_{1} w\right)\right|$. 
It is important to note that Lemma 1 covers linear timeinvariant systems as in this case $\psi(y)=0$. In other words, the proposed approach can always be applied to stabilizable and detectable linear time-invariant systems.

\section{ILLUSTRATIVE EXAMPLE}

In this section, we provide an example of a nonlinear system, which is not globally Lipschitz contrary to the systems addressed in Section V, to which our results apply. The control system consists of two coupled plants $P_{1}$ and $P_{2}$, whose origin is unstable, as in Section VII.B in [4]. The plants $P_{i}$, $i \in\{1,2\}$, are modeled as

$$
\begin{aligned}
& P_{1}:\left\{\begin{array}{l}
\dot{x}_{1}=d_{1} x_{1}^{2}-x_{1}^{3}+x_{2}+u_{1}+w \\
y_{1}=x_{1}
\end{array}\right. \\
& P_{2}:\left\{\begin{array}{l}
\dot{x}_{2}=d_{2} x_{2}^{2}-x_{2}^{3}+x_{1}+u_{2}+w \\
y_{2}=x_{2}
\end{array}\right.
\end{aligned}
$$

where $x_{i} \in \mathbb{R}, i \in\{1,2\}$, is the state of subsystem $P_{i}, y_{i}=x_{i}$ is its output, $d_{1}, d_{2} \in \mathbb{R}$ are unknown uncertain parameters (potentially time-varying) verifying $\left|d_{1}\right| \leq 1$ and $\left|d_{2}\right| \leq 1$, $w \in \mathbb{R}$ is the exogenous disturbance. For each subsystem, its own controller is collocated with the actuator and is given by $u_{i}=-2 y_{i}$.

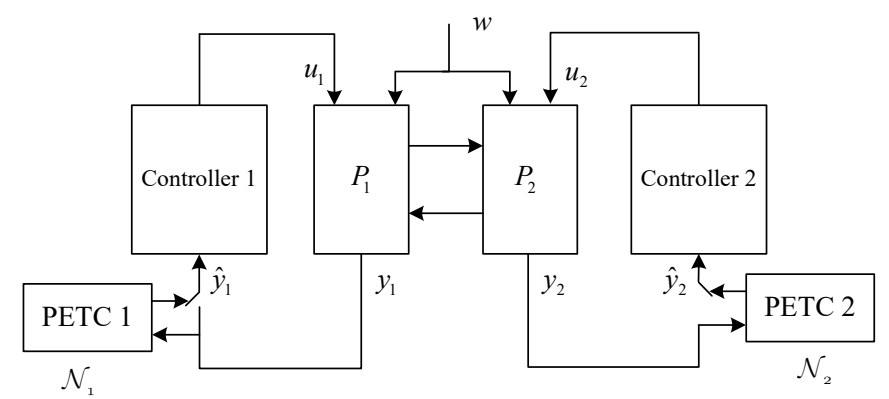

Fig. 2: Control setup of the two coupled systems.

We consider the case where the output measurements of $y_{1}$ and $y_{2}$ are respectively transmitted via two independently operating networks, $\mathcal{N}_{1}$ and $\mathcal{N}_{2}$, and received by the controller as $\hat{y}_{1}$ and $\hat{y}_{2}$, as illustrated in Figure 2. Zero-order-hold devices are used to implement the controller and this gives $\hat{f}_{v}=0$, as defined after (4). Let $e_{1}=\hat{y}_{1}-y_{1}$ and $e_{2}=\hat{y}_{2}-y_{2}$ be the networked-induced error (there is no need to introduce $\hat{u}-u$ since the controller is static), $x=\left(x_{1}, x_{2}\right), e=\left(e_{1}, e_{2}\right)$. Let $\tau=\left(\tau_{1}, \tau_{2}\right)$ with $\tau_{1}, \tau_{2} \in \mathbb{R}_{\geq 0}$. Note that $\kappa \in \mathbb{Z}_{>0}$ in (9) is irrelevant here since both networks have only one node. We obtain system (9) with $q=(x, e, \tau), F(q, w)=$ $(f(e, x, w), g(e, x, w), 1,1), f(x, e, w)=\left(d_{1} x_{1}^{2}-x_{1}^{3}+x_{2}-\right.$ $\left.2\left(x_{1}+e_{1}\right)+w, d_{2} x_{2}^{2}-x_{2}^{3}+x_{1}-2\left(x_{2}+e_{2}\right)+w\right), g(x, e, w)=$ $-f(x, e, w), C, D$ defined as after (9), $G(q)$ in (12) with $h_{i}\left(e_{i}, x\right)$ being defined as $h_{i}\left(e_{i}, x\right)=\left(1-\Gamma_{i}\left(e_{i}, x\right)\right) e_{i}$, $i \in\{1,2\}$.

We now verify Assumptions 1 and 2 . Let $i \in\{1,2\}$, we take $W_{i}\left(e_{i}\right)=\left|e_{i}\right|$. Assumption 1 holds with $\underline{\alpha}_{W_{i}}(s)=\bar{\alpha}_{W_{i}}(s)=$ $s$ for all $s \geq 0, \rho_{i}=0$ (since the networks consist of one node), $L_{W_{i}}=2, \widetilde{H}_{i}(x, e, w)=\left|-d_{i} x_{i}^{2}+x_{i}^{3}-x_{3-i}+2 x_{i}\right|+|w|$, $i \in\{1,2\}$.

To verify Assumption 2, we take $\delta_{i}\left(v_{i}\right)=0.5 y_{i}^{2}$ and consider the candidate Lyapunov function $V(x)=$ $a^{2} \sum_{i=1}^{2}\left(b \frac{x_{i}^{2}}{2}+c \frac{x_{i}^{4}}{4}\right)$ for any $x_{i} \in \mathbb{R}$, some $a, b, c>0$ and $i \in\{1,2\}$. Since $\left\langle\nabla \delta_{i}\left(v_{i}\right), f_{v_{i}}(x, e, w)\right\rangle \leq-2 x_{i}^{2}+$ $\left|d_{i} x_{i}^{3}+x_{i} x_{3-i}-2 x_{i} e_{i}+x_{i} w\right| \leq-4 \delta_{i}\left(v_{i}\right)+\mid d_{i} x_{i}^{3}-x_{i}^{4}+$ $\left.x_{i} x_{3-i}|+| 2 x_{i}^{2}|+| e_{i}\right|^{2}+|w|^{2} \leq L_{\delta_{i}} \delta_{i}\left(v_{i}\right)+J_{i}(x, e, w)$, item (iii) of Assumption 2 holds with $L_{\delta_{i}}=-4, J_{i}(x, e, w)=$ $\left|x_{i}^{3}+x_{i} x_{3-i}\right|+\left|2 d_{i} x_{i}^{2}\right|+\left|e_{i}^{2}\right|+|w|^{2}, i \in\{1,2\}$. We now verify item (ii) of Assumption 2. Since $2 s_{1} s_{2} \leq s_{1}^{2}+s_{2}^{2}$ for any $s_{1}, s_{2} \in \mathbb{R}$, we have that

$$
\begin{aligned}
& \langle\nabla V(x), f(x, e, w)\rangle \leq a^{2}\left(\sum _ { i = 1 } ^ { 2 } \left[\left(b^{2}+c^{2}\right) e_{i}^{2}+0.5\left(b^{2}+c^{2}\right) w^{2}\right.\right. \\
& \quad+(-c+1.5) x_{i}^{6}+c d_{i} x_{i}^{5}+(-b-2 c) x_{i}^{4}+b d_{i} x_{i}^{3} \\
& \left.\left.+(-2 b+1.5) x_{i}^{2}\right]+2 b x_{1} x_{2}+c x_{1}^{3} x_{2}+c x_{1} x_{2}^{3}\right) .
\end{aligned}
$$

Noting that $\left(s_{1}+s_{2}\right)^{2} \leq 2\left(s_{1}^{2}+s_{2}^{2}\right)$ for any $s_{1}, s_{2} \in \mathbb{R}$, $\widetilde{H}_{i}^{2}(x, e, w) \leq 2\left(-d_{i} x_{i}^{2}+x_{i}^{3}-x_{3-i}+2 x_{i}\right)^{2}+2 w^{2} \leq$ $4\left(-d_{i} x_{i}^{2}+x_{i}^{3}+2 x_{i}\right)^{2}+4 x_{3-i}^{2}+2 w^{2} \leq 4\left(x_{i}^{6}-2 d_{i} x_{i}^{5}+5 x_{i}^{4}-\right.$ $\left.4 d_{i} x_{i}^{3}+4 x_{i}^{2}\right)+4 x_{3-i}^{2}+2 w^{2}$ and $J_{i}(x, e, w) \leq 0.5 x_{i}^{2}+0.5\left(x_{i}^{2}+\right.$ $\left.x_{3-i}\right)^{2}+2 d_{i} x_{i}^{2}+e_{i}^{2}+w^{2} \leq x_{i}^{4}+x_{3-i}^{2}+\left(2 d_{i}+0.5\right) x_{i}^{2}+e_{i}^{2}+w^{2}$, $i \in\{1,2\}$. We then subtract and add $\widetilde{\alpha}(x, e, w)=\nu x^{2}+\nu e^{2}$, $\delta_{i}\left(v_{i}\right)=0.5 x_{i}^{2}, \widetilde{H}_{i}^{2}(x, e, w)$ and $J_{i}(x, e, w)$ to get

$$
\begin{aligned}
& \langle\nabla V(x), f(x, e, w)\rangle \leq-\alpha_{V}(x)-\alpha_{W}(e)+a^{2} p(x) \\
& +\sum_{i=1}^{2}\left[\varrho_{V_{i}}^{2}(|w|)-\delta_{i}\left(v_{i}\right)-\widetilde{H}_{i}^{2}(x, e, w)\right] \\
& +\sum_{i=1}^{2}\left[-J_{i}(x, e, w)+a^{2}\left(b^{2}+c^{2}+a^{-2}(1+\nu)\right) e_{i}^{2}\right],
\end{aligned}
$$

where $p(x):=\sum_{i=1}^{2} x_{i}^{2}\left[\left(-2 b+1.5+a^{-2}\left(21+2 d_{i}+\nu\right)\right)+d_{i}(b-\right.$ $\left.16 a^{-2}\right) x_{i}+\left(-b-2 c+21 a^{-2}\right) x_{i}^{2}+d_{i}\left(c-8 a^{-2}\right) x_{i}^{3}+(-c+$ $\left.\left.1.5+4 a^{-2}\right) x_{i}^{4}\right]+2 b x_{1} x_{2}+c x_{1}^{3} x_{2}+c x_{1} x_{2}^{3}, \varrho_{V_{i}}(s):=\left(0.5\left(b^{2}+\right.\right.$ $\left.\left.c^{2}\right)+3 a^{-2}\right) s^{2}$ for all $s \geq 0$. This implies that item (ii) of Assumption 2 holds with $\gamma_{1}=\gamma_{2}=a \sqrt{b^{2}+c^{2}+a^{-2}(1+\nu)}$ provided parameters $a, b, c, \nu$ are such that $p(x) \leq 0$ for all $x \in \mathbb{R}^{2}$. We take $(a, b, c, \nu)=(1.7,3.93,2.9,0.01)$ to ensure $p(x) \leq 0$ for all $x \in \mathbb{R}^{2}$, which yields $\gamma_{i}=8.36$ and determines the expression of $V(x)$, hence, item (i) of Assumption 2 holds.

Note that $\widetilde{H}_{i}(x, e, w)=H_{i}(x, e)+\varrho_{W_{i}}(|w|)$ with $H_{i}(x, e):=\left|-d_{i} x_{i}^{2}+x_{i}^{3}-x_{3-i}+2 x_{i}\right|$ and $\varrho_{W_{i}}(|w|):=|w|$ for $i \in\{1,2\}$, and $\widetilde{\alpha}_{V}(x, e, w)=\alpha_{V}(|x|)+\alpha_{W}(|e|)$ with $\alpha_{V}(|x|):=\nu|x|^{2}, \alpha_{W}(|e|):=\nu|e|^{2}$. Items 1) and 2) of Theorem 1 are therefore verified. We have that $\lambda_{i}^{*}=0.2289$ according to (14), from which we derive $T_{\mathrm{MASP}, i}\left(\lambda_{i}\right)$ for any $\lambda_{i} \in\left[0, \lambda_{i}^{*}\right)$. Indeed, $T_{\mathrm{MASP}, 1}$ can be taken as a function of $\lambda_{1}$, which tends to zero as $\lambda_{1}$ tends to its maximal value $\lambda_{1}^{*}$, and the maximal value for $T_{\mathrm{MASP}, 1}$ is 0.1634 , which arises when $\lambda_{1} \rightarrow 0$. As a result, the set $\mathcal{A}$ is ISS according to Theorem 1. 
TABLE II: Average inter-transmission time for $\mathcal{N}_{1}$ and $\mathcal{N}_{2}$ networks

\begin{tabular}{|c|c|c|c|c|c|c|c|}
\hline \multirow{2}{*}{} & \multicolumn{7}{|c|}{ Average inter-transmission time } \\
\cline { 3 - 8 } & $\lambda_{1}=0.06$ & $\lambda_{2}=0.02$ & $\lambda_{1}=0.1$ & $\lambda_{2}=0.09$ & $\lambda_{1}=0.15$ & $\lambda_{2}=0.12$ \\
\cline { 2 - 8 } & $\mathcal{N}_{1}$ & $\mathcal{N}_{2}$ & $\mathcal{N}_{1}$ & $\mathcal{N}_{2}$ & $\mathcal{N}_{1}$ & $\mathcal{N}_{2}$ \\
\hline$T_{1}=0.01$ & $T_{2}=0.02$ & 0.068 & 0.0403 & 0.0793 & 0.0781 & 0.0952 & 0.08 \\
\hline$T_{1}=0.04$ & $T_{2}=0.05$ & 0.0971 & 0.0556 & 0.101 & 0.098 & $\times$ & $\times$ \\
\hline$T_{1}=0.08$ & $T_{2}=0.1$ & 0.1103 & 0.101 & $\times$ & $\times$ & $\times$ & $\times$ \\
\hline
\end{tabular}

To illustrate the impact of $\lambda_{i}$ and sampling period $T_{i}$, $i \in\{1,2\}$, on the number of transmissions over the networks, we have considered different values of $\lambda_{i}$ and $T_{i}$ with $T_{i}<T_{\mathrm{MASP}, i}\left(\lambda_{i}\right)$ being satisfied, where $T_{\mathrm{MASP}, i}\left(\lambda_{i}\right)$ is the MASP determined by the given $\lambda_{i} \in\left(0, \lambda_{i}^{*}\right)$. We have set $\varepsilon_{i}=T_{i}$ for all $i \in\{1,2\}$, and run 50 simulations over 10 seconds with parameters $d_{1}=d_{2}=0.8$ and initial conditions randomly selected in $[-20,20]$ for both systems. Parameter $\varepsilon_{i}$ was selected as $T_{i}$, so that the triggering generators periodically evaluate their triggering condition. We have taken $w(t)=2 \sin (20 \pi t)$. The obtained average inter-transmission times over the 50 simulations are reported in Table II.

Empty boxes in Table II mean that the condition $T_{i}<$ $T_{\mathrm{MASP}, i}\left(\lambda_{i}\right)$ is violated. In view of the lines of Table II, we see that the average inter-transmission times increase when $\lambda_{i}$ grows for the same sampling period $T_{i}$. Also, when we keep the same triggering parameter $\lambda_{i}$ and vary the sampling period $T_{i}$, the average inter-transmission times increase with $T_{i}$. This suggests that, for this example and this set of simulations, setting sampling periods close to $T_{\mathrm{MASP}, i}\left(\lambda_{i}\right)$ uses less network bandwidth and ensures system stability. Interestingly, selecting $T_{i}$ large and $\lambda_{i}$ small, or $T_{i}$ small and $\lambda_{i}$ large, lead to similar average inter-transmission times in view of Table II.

\section{CONCLUSIONS}

We considered periodic event-triggered control of nonlinear systems subject to exogenous disturbances, where the controller communicates with the plant via multiple asynchronously operating networks. An emulation-based systematic design procedure was proposed, which is applicable for output feedback control. The starting point of the design is the availability of a controller, which robustly stabilizes the system in the absence of communication constraints. In the next step, the implementation of the controller over the networks was considered. Each network consists of multiple nodes, in which case a protocol is used to schedule transmissions. Moreover, a transmission over each network is triggered when a criterion, which only depends on the local measurements and the local control signals, is violated at given discrete sampling instants. We derived a hybrid system model to describe the resulting dynamics of the NCS and constructed a novel hybrid Lyapunov function for stability analysis. We provided conditions on the controller and scheduling protocols in order to design the local event-triggering criteria and explicit bounds on the maximum allowable sampling periods (MASP), to ensure input-to-state stability and $\mathcal{L}_{p}$-stability of the NCS. We showed that our design framework is applicable to a class of globally Lipschitz nonlinear systems and formulated the required conditions as linear matrix inequalities. We also showed explicitly that our results are applicable to any stabilizable and detectable linear time-invariant system. The effectiveness of the scheme was illustrated via simulations for a nonlinear example, which is not globally Lipschitz and suffered from parametric uncertainties.

Several extensions can be envisioned based on the framework laid down in this paper. Refined results could be developed for more specific classes of nonlinear systems. The results on LTI systems in Section V may also serve as a basis to derive co-design techniques, where both the triggering generator and the controller are designed simultaneously, similarly to [30] where CETC is studied.

\section{APPENDiX A PROOFS}

\section{A. Proof of Theorem 1}

We define, for any $q \in C \cup D$, the Lyapunov function

$$
\begin{aligned}
U(q) & :=V(x)+\sum_{i=1}^{N} S_{i}(q) \\
S_{i}(q) & :=\max \left\{\gamma_{i} \phi_{i}\left(\tau_{i}\right) W_{i}^{2}\left(e_{i}, \kappa_{i}\right), \lambda_{i} \delta_{i}\left(v_{i}\right)\right\},
\end{aligned}
$$

where $W_{i}, \delta_{i}$ and $V$ come from Assumptions 1 and 2, and $\phi_{i}:\left[0, T_{i}\right] \rightarrow\left[\underline{\mu}_{i}, \bar{\mu}_{i}\right]$ with $\bar{\mu}_{i}>\underline{\mu}_{i}>0$ is defined as in Lemma 3 in Appendix B.

We first show that the following properties hold for system (9). There exist $\bar{\alpha}_{U}, \underline{\alpha}_{U}, \alpha_{U}, \theta_{F} \in \mathcal{K}_{\infty}$ such that:

a) $U$ is locally Lipschitz in $x, e$ and $\tau$, and, for all $q \in C \cup D$, $\underline{\alpha}_{U}\left(|q|_{\mathcal{A}}\right) \leq U(q) \leq \bar{\alpha}_{U}\left(|q|_{\mathcal{A}}\right)$

b) for all $q \in C$ and $w \in \mathbb{R}^{n_{w}}, U^{\circ}(q ; F(q, w)) \leq$ $-\alpha_{U}(U(q))+\theta_{F}(|w|)$

c) for all $q \in D, w \in \mathbb{R}^{n_{w}}$ and $\mathrm{g} \in G(q), U(\mathrm{~g}) \leq U(q)$.

Proof of item a). It follows from Assumptions 1 and 2, the definition of $\phi_{i}$ in Lemma 3 that the Lipschitz property of $U$ in item a) is satisfied. Since $\delta_{i}$ is continuous and positive semidefinite, $v_{i}=g_{v_{i}}(x)$ with $g_{v_{i}}$ in (8) is continuous and $g_{v_{i}}(0)=$ 0 , as $g=\left(g_{p}, g_{c}\right)$ and $g_{p}(0)=g_{c}(0)=0$ with $g_{p}$ and $g_{c}$ in (1) and (2), there exists $\bar{\alpha}_{\delta_{i}} \in \mathcal{K}_{\infty}$ such that $\delta_{i}\left(v_{i}\right) \leq \bar{\alpha}_{\delta_{i}}(|x|)$, see Lemma 4.3 in [31]. In view of Lemma $3, \phi_{i}\left(\tau_{i}\right) \in\left[\underline{\mu}_{i}, \bar{\mu}_{i}\right]$ for all $\tau_{i} \in\left[0, T_{i}\right]$ with $0<\underline{\mu}_{i}^{*}<\underline{\mu}_{i}<\bar{\mu}_{i}<\bar{\mu}_{i}^{*}, i \in \bar{N}$. Consequently, in view of items (i) of Assumptions 1 and 2 and (27), $\underline{\alpha}_{V}(|x|)+\sum_{i=1}^{N} \gamma_{i} \underline{\mu}_{i} \underline{\alpha}_{W_{i}}^{2}(|e|) \leq U(q) \leq \bar{\alpha}_{V}(|x|)+$ $\sum_{i=1}^{N}\left(\gamma_{i} \bar{\mu}_{i} \bar{\alpha}_{W_{i}}^{2}(|e|)+\lambda_{i} \bar{\alpha}_{\delta_{i}}(|x|)\right)$. By applying Lemma 4 in Appendix B, we derive that there exist $\underline{\alpha}_{U}, \bar{\alpha}_{U} \in \mathcal{K}_{\infty}$ such that $\underline{\alpha}_{U}\left(|q|_{\mathcal{A}}\right) \leq U(q) \leq \bar{\alpha}_{U}\left(|q|_{\mathcal{A}}\right)$, hence item a) holds. 
Proof of item b). Let $q \in C, w \in \mathbb{R}^{n_{w}}$ and $i \in$ $\bar{N}$. We distinguish three cases according to Lemma 2 in Appendix B: Case I) $\gamma_{i} \phi_{i}\left(\tau_{i}\right) W_{i}^{2}\left(e_{i}, \kappa_{i}\right)<\lambda_{i} \delta_{i}\left(v_{i}\right)$; Case II) $\gamma_{i} \phi_{i}\left(\tau_{i}\right) W_{i}^{2}\left(e_{i}, \kappa_{i}\right)>\lambda_{i} \delta_{i}\left(v_{i}\right)$; and Case III) $\gamma_{i} \phi_{i}\left(\tau_{i}\right) W_{i}^{2}\left(e_{i}, \kappa_{i}\right)=\lambda_{i} \delta_{i}\left(v_{i}\right)$. Suppose that Cases I), II) and III), respectively, hold for $i \in N_{I}, i \in N_{I I}$ and $i \in N_{I I I}$, where $N_{I}, N_{I I}, N_{I I I} \subseteq \bar{N}$ and $N_{I} \cup N_{I I} \cup N_{I I I}=\bar{N}$. Then, in view of item (ii) of Assumption 2, items 1)-2) of Theorem 1 ,

$$
\begin{aligned}
U^{\circ}(q ; F(q, w)) & \leq-\alpha_{V}(|x|)-\alpha_{W}(|e|) \\
& +\sum_{i \in \bar{N}}\left(Z_{i}(q, w)+S_{i}^{\circ}(q ; F(q, w))\right),
\end{aligned}
$$

where $Z_{i}(q, w)=\gamma_{i}^{2} W_{i}^{2}\left(e_{i}\right)-\widetilde{H}_{i}^{2}(x, e, w)-J_{i}(x, e, w)-$ $\delta_{i}\left(v_{i}\right)+\varrho_{V, i}(|w|)$. We next consider $S_{i}^{\circ}(q ; F(q, w))$ for $i \in N_{I}$, $i \in N_{I I}$ and $i \in N_{I I I}$, respectively.

Case I) $i \in N_{I}$. We have that $S_{i}(q)=\lambda_{i} \delta_{i}\left(v_{i}\right)$ in (27) in this case. It then follows from item (iii) of Assumption 2 and [32] that

$S_{i}^{\circ}(q ; F(q, w)) \leq \lambda_{i}\left(L_{\delta_{i}} \delta_{i}\left(v_{i}\right)+\widetilde{H}_{i}^{2}(x, e, w)+J_{i}(x, e, w)\right)$.

Since $\phi_{i}\left(\tau_{i}\right) \geq \underline{\mu}_{i}>\underline{\mu}_{i}^{*}=\bar{\rho}_{i} \geq \frac{\gamma_{i} \lambda_{i}}{1-\lambda_{i} L_{\delta_{i}}}$ according to Lemma 3, $1-\lambda_{i} L_{\delta_{i}}>0$ as ensured by $\lambda_{i}<\lambda_{i}^{*}$ with $\lambda_{i}^{*}$ defined in (14), $\gamma_{i} \phi_{i}\left(\tau_{i}\right) W_{i}^{2}\left(e_{i}, \kappa_{i}\right)<\lambda_{i} \delta_{i}\left(v_{i}\right)$ implies that $\gamma_{i}^{2} W_{i}^{2}\left(e_{i}, \kappa_{i}\right)<\frac{\gamma_{i} \lambda_{i} \delta_{i}\left(v_{i}\right)}{\phi_{i}\left(\tau_{i}\right)}<\frac{\gamma_{i} \lambda_{i} \delta_{i}\left(v_{i}\right)}{\bar{\rho}_{i}} \leq \frac{\gamma_{i} \lambda_{i} \delta_{i}\left(v_{i}\right)}{\frac{\gamma_{i} \lambda_{i}}{1-\lambda_{i} L_{\delta_{i}}}}=$ $\left(1-\lambda_{i} L_{\delta_{i}}\right) \delta_{i}\left(v_{i}\right)$, and

$$
\begin{aligned}
& Z_{i}(q, w)+S_{i}^{\circ}(q ; F(q, w)) \leq \gamma_{i}^{2} W_{i}^{2}\left(e_{i}\right)-\widetilde{H}_{i}^{2}(x, e, w) \\
& \quad-J_{i}(x, e, w)-\delta_{i}\left(v_{i}\right)+\varrho_{V, i}(|w|) \\
& \quad+\lambda_{i}\left(L_{\delta_{i}} \delta_{i}\left(v_{i}\right)+\widetilde{H}_{i}^{2}(x, e, w)+J_{i}(x, e, w)\right) \\
& \leq-\left(1-\lambda_{i}\right)\left(\widetilde{H}_{i}^{2}(x, e, w)+J_{i}(x, e, w)\right)+\varrho_{V, i}(|w|) \\
& \leq \varrho_{V, i}(|w|)
\end{aligned}
$$

since $\lambda_{i}<\lambda_{i}^{*} \leq 1$ in view of (14).

Case II) $i \in N_{I I}$. We have that $S_{i}(q)=\gamma_{i} \phi_{i}\left(\tau_{i}\right) W_{i}^{2}\left(e_{i}, \kappa_{i}\right)$ in this case. We omit below the dependency of $\phi_{i}$ on $\tau_{i}$ for the sake of convenience. In view of item (ii) in Assumption 1 and the facts that $\phi_{i}\left(\tau_{i}\right) \leq \bar{\mu}_{i}$ according to Lemma 3, $\widetilde{H}_{i}(x, e, w)=H_{i}(x, e)+\varrho_{W_{i}}(|w|)$ according to item 1) of Theorem 1, $\sqrt{s_{1}^{2}+s_{2}^{2}} \leq s_{1}+s_{2}$ and $2 s_{3} s_{4} \leq \nu s_{3}^{2}+\frac{1}{\nu} s_{4}^{2}$, for $s_{1}, s_{2}, s_{3}, s_{4} \geq 0$ and $\nu>0$,

$S_{i}^{\circ}(q ; F(q, w))=\gamma_{i}\left(-2\left(L_{W_{i}}+\tilde{\nu}_{i}\right) \phi_{i}-\gamma_{i}\left(\phi_{i}^{2}+1\right)\right) W_{i}^{2}\left(e_{i}, \kappa_{i}\right)$

$+2 \gamma_{i} \phi_{i} W_{i}\left(e_{i}, \kappa_{i}\right)\left(L_{W_{i}} W_{i}\left(e_{i}, \kappa_{i}\right)+H_{i}(x, e)+\varrho_{W_{i}}(|w|)\right)$

$\leq \gamma_{i}\left(-\left(2 L_{W_{i}}+\tilde{\nu}_{i}\right) \phi_{i}-\gamma_{i}\left(\phi_{i}^{2}+1\right)\right) W_{i}^{2}\left(e_{i}, \kappa_{i}\right)$

$+2 \gamma_{i} L_{W_{i}} \phi_{i} W_{i}^{2}\left(e_{i}, \kappa_{i}\right)+\gamma_{i}^{2} \phi_{i}^{2} W_{i}^{2}\left(e_{i}, \kappa_{i}\right)+H_{i}^{2}(x, e)$

$+\gamma_{i} \phi_{i}\left(\tilde{\nu}_{i} W_{i}^{2}\left(e_{i}, \kappa_{i}\right)+\frac{1}{\tilde{\nu}_{i}} \varrho_{W_{i}}^{2}(|w|)\right)$

$\leq-\gamma_{i}^{2} W_{i}^{2}\left(e_{i}, \kappa_{i}\right)+H_{i}^{2}(x, e)+\frac{\bar{\mu}_{i} \gamma_{i}}{\tilde{\nu}_{i}} \varrho_{W_{i}}^{2}(|w|)$, where $\tilde{\nu}_{i}>0$ is given in Lemma 3. Then, since $\bar{\mu}_{i}<\bar{\mu}_{i}^{*}$ and $\widetilde{H}_{i}^{2}(x, e, w) \geq H_{i}^{2}(x, e)$,

$$
\begin{aligned}
& Z_{i}(q, w)+S_{i}^{\circ}(q ; F(q, w)) \leq \gamma_{i}^{2} W_{i}^{2}\left(e_{i}\right)-\widetilde{H}_{i}^{2}(x, e, w) \\
& \quad-J_{i}(x, e, w)-\delta_{i}\left(v_{i}\right)+\varrho_{V, i}(|w|) \\
& \quad-\gamma_{i}^{2} W_{i}^{2}\left(e_{i}, \kappa_{i}\right)+H_{i}^{2}(x, e)+\frac{\bar{\mu}_{i} \gamma_{i}}{\tilde{\nu}_{i}} \varrho_{W_{i}}^{2}(|w|) \\
& \leq-\delta_{i}\left(v_{i}\right)-J_{i}(x, e, w)+\frac{\bar{\mu}_{i} \gamma_{i}}{\tilde{\nu}_{i}} \varrho_{W_{i}}^{2}(|w|)+\varrho_{V, i}(|w|) \\
& \leq \frac{\bar{\mu}_{i}^{*} \gamma_{i}}{\tilde{\nu}_{i}} \varrho_{W_{i}}^{2}(|w|)+\varrho_{V, i}(|w|) .
\end{aligned}
$$

Case III) $i \in N_{I I I}$. In view of Lemma 2 and (30), in this case, $Z_{i}(q, w)+S_{i}^{\circ}(q ; F(q, w)) \leq \frac{\bar{\mu}_{i}^{*} \gamma_{i}}{\tilde{\nu}_{i}} \varrho_{W_{i}}^{2}(|w|)+\varrho_{V, i}(|w|)$.

In view of Cases I)-III),

$$
\begin{aligned}
& U^{\circ}(q ; F(q, w)) \leq-\alpha_{V}(|x|)-\alpha_{W}(|e|) \\
& \quad+\sum_{i \in \bar{N}}\left(Z_{i}(q, w)+S_{i}^{\circ}(q ; F(q, w))\right) \\
& \leq-\alpha_{V}(|x|)-\alpha_{W}(|e|)+\sum_{i \in \bar{N}}\left(\frac{\bar{\mu}_{i}^{*} \gamma_{i}}{\tilde{\nu}_{i}} \varrho_{W_{i}}^{2}(|w|)+\varrho_{V, i}(|w|)\right) .
\end{aligned}
$$

In view of item a), there exists $\alpha_{U} \in \mathcal{K}_{\infty}$ such that item b) holds with $\theta_{F}(s):=\sum_{i \in \bar{N}} \theta_{F_{i}}(s)$, and $\theta_{F_{i}}(s):=\frac{\bar{\mu}_{i}^{*} \gamma_{i}}{\tilde{\nu}_{i}} \varrho_{W_{i}}^{2}(s)+\varrho_{V, i}(s)$ for all $s \geq 0$.

Proof of item c). Let $q \in D_{i}, i \in \bar{N}$. We distinguish two cases whether a transmission occurs. When a transmission occurs, $W_{i}\left(\chi_{i}\left(e_{i}, \kappa_{i}\right), \kappa_{i}+1\right) \leq \rho_{i} W_{i}\left(e_{i}, \kappa_{i}\right)$ according to item (ii) of Assumption 1. Note that $\phi_{i}(0)<\bar{\mu}_{i}^{*}=1 / \bar{\rho}_{i}, \phi_{i}\left(T_{i}\right)>\underline{\mu}_{i}^{*}=$ $\bar{\rho}_{i}$ and $\bar{\rho}_{i} \geq \rho_{i}$ in view of Lemma 3. Let $g \in \mathcal{G}_{i}(q)$,

$$
\begin{aligned}
S_{i}(g) & \left.=\max \left\{\gamma_{i} \phi_{i}(0) W_{i}^{2}\left(\chi_{i}\left(e_{i}, \kappa_{i}\right), \kappa_{i}+1\right)\right), \lambda_{i} \delta_{i}\left(v_{i}\right)\right\} \\
& \leq \max \left\{\gamma_{i} \frac{1}{\bar{\rho}_{i}} \rho_{i}^{2} W_{i}^{2}\left(e_{i}, \kappa_{i}\right), \lambda_{i} \delta_{i}\left(v_{i}\right)\right\} \\
& \leq \max \left\{\gamma_{i} \bar{\rho}_{i} W_{i}^{2}\left(e_{i}, \kappa_{i}\right), \lambda_{i} \delta_{i}\left(v_{i}\right)\right\} \\
& \leq \max \left\{\gamma_{i} \phi_{i}\left(T_{i}\right) W_{i}^{2}\left(e_{i}, \kappa_{i}\right), \lambda_{i} \delta_{i}\left(v_{i}\right)\right\} \leq S_{i}(q) .
\end{aligned}
$$

When no transmission occurs, it follows from (7), (13) and item (ii) of Assumption 1 that $\gamma_{i} W_{i}^{2}\left(\chi_{i}\left(e_{i}, \kappa_{i}\right), \kappa_{i}\right)=$ $\gamma_{i} W_{i}^{2}\left(e_{i}, \kappa_{i}\right) \leq \lambda_{i} \bar{\rho}_{i} \delta_{i}\left(v_{i}\right)$. Since $\phi_{i}(0)<\bar{\mu}_{i}^{*}=1 / \bar{\rho}_{i}$ according to Lemma 3 , we have that

$$
\begin{aligned}
S_{i}(g) & =\max \left\{\gamma_{i} \phi_{i}(0) W_{i}^{2}\left(e_{i}, \kappa_{i}\right), \lambda_{i} \delta_{i}\left(v_{i}\right)\right\} \\
& \leq \max \left\{\frac{1}{\bar{\rho}_{i}} \lambda_{i} \bar{\rho}_{i} \delta_{i}\left(v_{i}\right), \lambda_{i} \delta_{i}\left(v_{i}\right)\right\}=\lambda_{i} \delta_{i}\left(v_{i}\right) \leq S_{i}(q) .
\end{aligned}
$$

As a result, for all $q \in D$ and $g \in G(q)$,

$$
U(g) \leq V(x)+\sum_{i=1}^{N} S_{i}(g) \leq V(x)+\sum_{i=1}^{N} S_{i}(q)=U(q),
$$

and item c) holds.

Let $(\varphi, w)$ be a solution to (9). Note that $U$ is locally Lipschitz in $x, e, \tau$ from item a). In view of page 99 in [32], for 
any $\nu \in(0,1)$, all $j \in \mathbb{Z}_{>0}$ such that there exists $t \in \mathbb{R}_{>0}$ with $(t, j) \in \operatorname{dom} \varphi$ and almost all $s \in I^{j}:=\{\bar{t}:(\bar{t}, j) \in \operatorname{dom} \phi\}$,

$$
\begin{aligned}
\frac{d}{d s} U(\varphi(s, j)) \leq & U^{\circ}(\varphi(s, j) ; F(\varphi(s, j), w(s, j))) \\
\leq & -(1-\nu) \alpha_{U}(U(\varphi(s, j)) \\
& -\nu \alpha_{U}\left(U(\varphi(s, j))+\theta_{F}(|w(s, j)|) .\right.
\end{aligned}
$$

By invoking standard ISS arguments, we derive that there exists $\bar{\beta} \in \mathcal{K} \mathcal{L}$ such that for all $(t, j) \in \operatorname{dom} \varphi$,

$U(\varphi(t, j)) \leq \bar{\beta}\left(U\left(\varphi\left(t, t_{j}\right), t-t_{j}\right)+\alpha_{U}^{-1}\left(\frac{1}{\nu} \theta_{F}\left(\|w\|_{(t, j)}\right)\right)\right.$,

where $t_{j}:=\inf I_{j}$. It follows from item c) that, for all $j$ such that $(t, j) \in \operatorname{dom} \varphi$,

$$
U\left(\varphi\left(t_{j+1}, j+1\right)\right) \leq U\left(\varphi\left(t_{j+1}, j\right)\right) .
$$

Let $\varepsilon:=\min _{i \in \bar{N}} \varepsilon_{i}$, where $\varepsilon_{i}>0$ is the minimum inter-sampling time corresponding to network $\mathcal{N}_{i}$. Let $(t, j) \in \operatorname{dom} \varphi$. The integer $j$ represents the total number of transmissions over the $N$ networks, we can therefore write it as $j=j_{1}+\ldots+j_{N}$, where $j_{i}$ is the number of transmissions that has occured so far on network $\mathcal{N}_{i}$. In view of the definition of the jump set in (9), $t \geq \varepsilon\left(j_{i}-1\right)$. Consequently, $\frac{t}{N} \geq \frac{\varepsilon}{N}\left(j_{i}-1\right)$ and $t=N \frac{t}{N} \geq \frac{\varepsilon}{N}\left(j_{1}+\ldots .+j_{N}\right)-\varepsilon=\frac{\varepsilon}{N} j-\varepsilon$. Since $t \geq 0$, we have that $t \geq t / 2+\varepsilon / 2 \max \left\{\frac{j}{N}-1,0\right\}$. Consequently, for any $(t, j) \in \operatorname{dom} \varphi$,

$$
\begin{aligned}
U(\varphi(t, j)) \leq & \bar{\beta}\left(U(\varphi(0,0), t)+\alpha_{U}^{-1}\left(\frac{1}{\nu} \theta_{F}\left(\|w\|_{(t, j)}\right)\right)\right. \\
\leq & \bar{\beta}\left(U(\varphi(0,0)), 0.5 t+0.5 \varepsilon \max \left\{\frac{j}{N}-1,0\right\}\right) \\
& +\alpha_{U}^{-1}\left(\frac{1}{\nu} \theta_{F}\left(\|w\|_{(t, j)}\right)\right) .
\end{aligned}
$$

Since $\alpha\left(s_{1}+s_{2}\right) \leq \alpha\left(2 s_{1}\right)+\alpha\left(2 s_{2}\right)$ for any $\alpha \in \mathcal{K}_{\infty}$ and $s_{1}, s_{2} \geq 0$, see (7) in [33], we deduce from item a), to have that $|\varphi(t, j)|_{\mathcal{A}} \leq \beta\left(|\varphi(0,0)|_{\mathcal{A}}, t+j\right)+\psi\left(\|w\|_{(t, j)}\right)$, for all $(t, j) \in \operatorname{dom} \varphi$, where $\beta\left(s_{1}, s_{2}\right) \quad:=$ $\underline{\alpha}_{U}^{-1}\left(2 \bar{\beta}\left(\bar{\alpha}_{U}\left(s_{1}\right), 0.5 \min \{\varepsilon, 1\} \max \left\{\frac{s_{2}}{N}-2,0\right\}\right)\right) \quad$ and $\psi(s):=\underline{\alpha}_{U}^{-1}\left(2 \alpha_{U}^{-1}\left(\frac{1}{\nu} \theta_{F}\left(\|w\|_{(t, j)}\right)\right)\right)$.

\section{B. Sketched proof of Theorem 2}

For any $q \in C \cup D$, let $U(q):=V(x)+$ $\max \left\{\gamma \phi(\tau) W^{2}(e, \kappa), \lambda V(x)\right\}$ with $W$ and $V$ coming from Assumption 3. The function $\phi:[0, T] \rightarrow[\underline{\mu}, \bar{\mu}]$ is defined as in Lemma 5 in Appendix B.

The proof Theorem 2 follows same steps as the proof of Theorem 1 . We only explicitly prove the flow property corresponding to item b) in Appendix A-A in the following. In particular, we need show that item $\mathrm{b}$ ) holds under Assumption 3. Let $q \in C$ and $w \in \mathbb{R}^{n_{w}}$ and we distinguish three cases according to Lemma 2 .
Case I) $\gamma \phi(\tau) W^{2}(e, \kappa)<\lambda V(x)$. We have that $U(q)=$ $(\lambda+1) V(x)$ in this case. According to Lemma 5, $\phi(\tau) \geq$ $\bar{\rho}>\frac{\gamma \lambda}{a_{V}}$. Hence, there exists $\nu \in(0,1)$ such that $\bar{\rho} \geq$ $\frac{1}{1-\nu} \frac{\gamma \lambda}{a_{V}}$. Thus, $\gamma^{2} W^{2}(e, \kappa) \leq \frac{\gamma \lambda V(x)}{\bar{\rho}} \leq(1-\nu) a_{V} V(x)$ and $U^{\circ}(q ; F(q, w)) \leq(\lambda+1)\left(-a_{V} V(x)-\alpha_{W}(|e|)+\right.$ $\left.\gamma^{2} W^{2}(e, \kappa)+\rho_{V}(|w|)\right) \leq(\lambda+1)\left(-\nu V(x)-\alpha_{W}(|e|)+\right.$ $\left.\varrho_{V}(|w|)\right)$.

Case II) $\gamma \phi(\tau) W(e, \kappa)>\lambda V(x)$. In this case, we have that $U(q)=V(x)+\gamma \phi(\tau) W^{2}(e, \kappa)$. From item (ii) of Assumption 3 , the facts that $\phi(\tau) \leq \bar{\mu}$ in view of Lemma 5 , and $2 a b \leq$ $c a^{2}+1 / c b^{2}$ for all $a, b \geq 0$ and $c>0$,

$$
\begin{aligned}
& U^{\circ}(q ; F(q, w))=-a_{V} V(x)-\alpha_{W}(|e|) \\
& +\gamma^{2} W^{2}(e, \kappa)+\rho_{V}(|w|) \\
& +\gamma\left(-\left(2 L_{W}+\tilde{\nu}\right) \phi-\gamma\left(\frac{L_{V}^{2}}{a_{V}-\tilde{\nu}} \phi^{2}+1\right)\right) W^{2}(e, \kappa) \\
& +2 \gamma \phi W(e, \kappa)\left(L_{W} W(e, \kappa)+L_{V} \sqrt{V(x)}+\varrho_{W}(|w|)\right) \\
& \leq-a_{V} V(x)-\alpha_{W}(|e|)+\gamma^{2} W^{2}(e, \kappa)+\rho_{V}(|w|) \\
& -\gamma\left(2 L_{W}+\tilde{\nu}\right) \phi W^{2}(e, \kappa)-\gamma^{2}\left(\frac{L_{V}^{2}}{a_{V}-\tilde{\nu}} \phi^{2}+1\right) W^{2}(e, \kappa) \\
& +\gamma^{2} \frac{L_{V}^{2}}{a_{V}-\tilde{\nu}} \phi^{2} W^{2}(e, \kappa)+\left(a_{V}-\tilde{\nu}\right) V(x) \\
& \quad+2 \gamma L_{W} \phi W^{2}(e, \kappa)+\gamma \phi\left(\tilde{\nu} W^{2}(e, \kappa)+\frac{1}{\tilde{\nu}} \varrho_{W}^{2}(|w|)\right) \\
& \leq-\tilde{\nu} V(x)-\alpha_{W}(|e|)+\frac{\bar{\mu} \gamma}{\tilde{\nu}} \varrho_{W}^{2}(|w|)+\varrho_{V}(|w|),
\end{aligned}
$$

where $\tilde{\nu}>0$ comes from Lemma 5.

Case III: $\gamma \phi(\tau) W^{2}(e, \kappa)=\lambda V(x)$. In view of Lemma 2, and cases I and II, $U^{\circ}(q ; F(q, w)) \leq-\min \{\nu, \tilde{\nu}\} V(x)-$ $\alpha_{W}(|e|)+\varrho_{V}(|w|)+\frac{\bar{\mu} \gamma}{\tilde{\nu}} \varrho_{W}^{2}(|w|)$.

In view of cases I)-III), $\quad U^{\circ}(q ; F(q, w)) \leq$ $-\min \{\nu, \tilde{\nu}\} V(x)-\alpha_{W}(|e|)+\varrho_{V}(|w|)+\frac{\bar{\mu} \gamma}{\tilde{\nu}} \varrho_{W}^{2}(|w|)$ for all $q \in C$ and $w \in \mathbb{R}^{n_{w}}$. Then, there exists $\alpha_{U} \in \mathcal{K}_{\infty}$ such that item b) holds with $\theta_{F}(s):=\frac{\bar{\mu} \gamma}{\tilde{\nu}} \varrho_{W}^{2}(|w|)+\varrho_{V}(|w|)$ for all $s \geq 0$. The rest of proof follows the same steps as the proof of Theorem 1 and is therefore omitted.

\section{Sketched proof of Theorem 3}

We define the storage function as (27) and prove the desired result by ensuring the satisfaction of the next properties.

a) $U$ is locally Lipschitz in $x, e$ and $\tau$, and, for all $q \in C \cup D$, $\underline{\alpha}_{U}\left(|q|_{\mathcal{A}}\right) \leq U(q) \leq \bar{\alpha}_{U}\left(|q|_{\mathcal{A}}\right)$, where $\bar{\alpha}_{U}, \underline{\alpha}_{U} \in \mathcal{K}_{\infty} ;$

b) for all $q \in C$ and $w \in \mathbb{R}^{n_{w}}, U^{\circ}(q ; F(q, w)) \leq \mu\left(\theta^{p}|w|^{p}-\right.$ $\left.|z|^{p}\right)$

c) for all $q \in D, w \in \mathbb{R}^{n_{w}}$ and $\mathrm{g} \in G(q), U(\mathrm{~g}) \leq U(q)$.

The proof of items a) and c) follows the same steps as the proof of Theorem 1. We therefore prove the flow property corresponding to item b) in Appendix A-A. Recall that there are three cases to consider when $q \in C$ : Case I) 
$\gamma_{i} \phi_{i}\left(\tau_{i}\right) W_{i}^{2}\left(e_{i}, \kappa_{i}\right)<\lambda_{i} \delta_{i}\left(v_{i}\right)$; Case II $) \gamma_{i} \phi_{i}\left(\tau_{i}\right) W_{i}^{2}\left(e_{i}, \kappa_{i}\right)>$ $\lambda_{i} \delta_{i}\left(v_{i}\right)$; and Case III) $\gamma_{i} \phi_{i}\left(\tau_{i}\right) W_{i}^{2}\left(e_{i}, \kappa_{i}\right)=\lambda_{i} \delta_{i}\left(v_{i}\right)$, and they are respectively hold for $i \in N_{I}, i \in N_{I I}$ and $i \in N_{I I I}$. We derive that the following hold.

Case I) $i \in N_{I}$. In this case, $S_{i}^{\circ}(q ; F(q, w)) \leq \lambda_{i}\left(L_{\delta_{i}} \delta_{i}\left(v_{i}\right)+\right.$ $\left.\widetilde{H}_{i}^{2}(x, e, w)+J_{i}(x, e, w)\right)$. Let $Z_{i}(q, w)=\gamma_{i}^{2} W_{i}^{2}\left(e_{i}\right)-$ $\widetilde{H}_{i}^{2}(x, e, w)-J_{i}(x, e, w)-\delta_{i}\left(v_{i}\right)$. We have that $Z_{i}(q, w)+$ $S_{i}^{\circ}(q ; F(q, w)) \leq \gamma_{i}^{2} W_{i}^{2}\left(e_{i}, \kappa_{i}\right)-\left(1-\lambda_{i} L_{\delta_{i}}\right) \delta_{i}\left(v_{i}\right)-(1-$ $\left.\lambda_{i}\right)\left(\widetilde{H}_{i}^{2}(x, e, w)+J_{i}(x, e, w)\right) \leq-\left(1-\lambda_{i}\right) \widetilde{H}_{i}^{2}(x, e, w)-$ $\left(1-\lambda_{i}\right) J_{i}(x, e, w), i \in \bar{N}$, since $\lambda_{i} L_{\delta_{i}}<1$ from (14) and $\gamma_{i}^{2} W_{i}^{2}\left(e_{i}, \kappa_{i}\right)<\left(1-\lambda_{i} L_{\delta_{i}}\right) \delta_{i}\left(v_{i}\right)$.

Case II) $i \in N_{I I}$. In Case II $), \quad S_{i}^{\circ}(q ; F(q, w))=$ $\gamma_{i}\left(-\left(2 L_{W_{i}}+\tilde{\nu}_{i}\right) \phi_{i}-\gamma_{i}\left(\phi_{i}^{2}+{ }^{1} 1\right)\right) W_{i}^{2}\left(e_{i}, \kappa_{i}\right)+$ $2 \gamma_{i} \phi_{i} W_{i}\left(e_{i}, \kappa_{i}\right)\left(L_{W_{i}} W_{i}\left(e_{i}, \kappa_{i}\right)+\widetilde{H}_{i}(x, e, w)\right) \leq$ $-\gamma_{i}^{2} W_{i}^{2}\left(e_{i}, \kappa_{i}\right)+\widetilde{H}_{i}^{2}(x, e, w)$ and $Z_{i}(q, w)+S_{i}^{\circ}(q ; F(q, w)) \leq$ $-\delta_{i}\left(v_{i}\right)-J_{i}(x, e, w), i \in \bar{N}$.

Case III) $i \in N_{I I I}$. In view of Lemma 2, and cases I and II, $Z_{i}(q, w)+S_{i}^{\circ}(q ; F(q, w)) \leq \max \left\{-\left(1-\lambda_{i}\right) \widetilde{H}_{i}^{2}(x, e, w)-\right.$ $\left.\left(1-\lambda_{i}\right) J_{i}(x, e, w),-\delta_{i}\left(v_{i}\right)-J_{i}(x, e, w)\right\}$.

Consequently, $\quad U^{\circ}(q ; F(q, w)) \leq \mu\left(\theta^{p}|w|^{p} \quad\right.$ $\left.|z|^{p}\right)+\sum_{i \in \bar{N}} Z_{i}(q, w)+S_{i}^{\circ}(q ; F(q, w)) \leq \mu\left(\theta^{p}|w|^{p}-\right.$ $\left.|z|^{p}\right) \quad-\sum_{i \in N_{I I}}\left(\delta_{i}\left(v_{i}\right)+J_{i}(x, e, w)\right)-\sum_{i \in N_{I}}(1-$ $\left.\lambda_{i}\right)\left(\widetilde{H}_{i}^{2}(x, e, w)+J_{i}(x, e, w)\right)-\sum_{i \in N_{I I I}} \max \{(1-$ $\left.\left.\lambda_{i}\right)\left(\widetilde{H}_{i}^{2}(x, e, w)+J_{i}(x, e, w)\right)+\delta_{i}\left(v_{i}\right)+J_{i}(x, e, w)\right\} \leq$ $\mu\left(\theta^{p}|w|^{p}-|z|^{p}\right)$ since $\lambda_{i}<\lambda_{i}^{*} \leq 1$ in view of (14). The satisfaction of items a) and c) follows by applying similar arguments as in the proof of Theorem 1.

Let $(\varphi, w)$ be a solution to (9). In view of page 99 in [32], for all $j \in \mathbb{Z}_{\geq 0}$ such that there exists $t \in \mathbb{R}_{>0}$ with $(t, j) \in \operatorname{dom} \varphi$ and almost all $s \in I^{j}:=\{\bar{t}:(\bar{t}, j) \in$ $\operatorname{dom} \phi\}, \frac{d}{d s} U(\varphi(s, j)) \leq U^{\circ}(\varphi(s, j) ; F(\varphi(s, j), w(s, j))) \leq$ $-|z(s, j)|^{p}+\theta^{p}|w(s, j)|^{p}$. We then follow similar lines as the the proof of Theorem 1 and the Theorem IV.7 in [26] and we obtain that $\|z\|_{\mathcal{L}_{p}} \leq \tilde{\gamma}\left(|\varphi(0,0)|_{\mathcal{A}}\right)+\theta\|w\|_{\mathcal{L}_{p}}$, where $\tilde{\gamma}:=\bar{\alpha}_{U}$.

\section{Proof of Proposition 1}

Let matrix $P, a_{V}, a_{W}, \tilde{\theta}, \epsilon_{i}>0$ and $\nu_{i} \geq a_{W}+\varpi_{i}^{2}\left|\overline{\mathcal{B}}_{2, i}\right|^{2}$, $i \in \bar{N}$, be given such that (24) holds. Let $i \in \bar{N}$, since $\left|\frac{\partial W_{i}\left(e_{i}, \kappa_{i}\right)}{\partial e_{i}}\right| \leq \varpi_{i}$ for almost all $e_{i} \in \mathbb{R}^{n_{e_{i}}}$ and $\kappa_{i} \in \mathbb{Z}_{\geq 0}$ (as the protocol is UGES, see discussions above (21)), we have that for all $x \in \mathbb{R}^{n_{x}}$ and almost all $e \in$ $\mathbb{R}^{n_{e}},\left\langle\frac{\partial W_{i}\left(e_{i}, \kappa_{i}\right)}{\partial e_{i}},-\bar{C}_{i}\left(\mathcal{A}_{1} x+\mathcal{B}_{1} e+\mathcal{D}_{1} \bar{\psi}(x)+\mathcal{E}_{1} w\right)\right\rangle \leq$ $\varpi_{i}\left|\bar{C}_{i} \mathcal{A}_{1} x+\bar{C}_{i} \mathcal{B}_{1} e+\bar{C}_{i} \mathcal{D}_{1} \bar{\psi}(x)+\bar{C}_{i} \mathcal{E}_{1} w\right| \leq \varpi_{i} \mid \bar{C}_{i} \mathcal{A}_{1} x+$ $\left.\overline{\mathcal{B}}_{2, i} e+\bar{C}_{i} \mathcal{D}_{1} \bar{\psi}(x)+\bar{C}_{i} \mathcal{E}_{1} w|+| \bar{C}_{i} \mathcal{B}_{1} \bar{\Psi}_{i} e \mid\right)$ and $\left|\bar{C}_{i} \mathcal{D}_{1} \bar{\psi}(x)\right| \leq$ $L\left|\bar{C}_{i} \mathcal{D}_{1}\right||x|$ in view of the properties of $\psi$. Note that $\overline{\mathcal{B}}_{2, i} e$ is independent to $e_{i}$ and $\mathcal{B}_{1} \bar{\Psi}_{i} e$ depends only on $e_{i}$ according to the definition of $\bar{B}_{2, i}$ and $\bar{\Psi}_{i}$. This implies that item (iii) of Assumption 1 holds with $L_{W_{i}}=\varpi_{i} / \underline{a}_{W_{i}}\left|\bar{C}_{i} \mathcal{B}_{1} \bar{\Psi}_{i}\right|$, $\widetilde{H}_{i}(x, e, w)=\varpi_{i}\left(\left|\bar{C}_{i} \mathcal{A}_{1} x+\overline{\mathcal{B}}_{2, i} e+\bar{C}_{i} \mathcal{E}_{1} w\right|+L\left|\bar{C}_{i} \mathcal{D}_{1}\right||x|\right)$.
Recall that $\delta_{i}\left(v_{i}\right)=\epsilon_{i}^{2}\left|v_{i}\right|^{2}, v_{i}=\bar{C}_{i} x$ and note that $\left|x^{T}\left(\bar{C}_{i}^{T} \bar{C}_{i} \mathcal{A}_{1}+\mathcal{A}_{1}^{T} \bar{C}_{i}^{T} \bar{C}_{i}\right) x\right|=2\left|\left\langle\bar{C}_{i} x, \quad \bar{C}_{i} \mathcal{A}_{1} x\right\rangle\right| \leq$ $2\left|\bar{C}_{i} x\right|\left|\bar{C}_{i} \mathcal{A}_{1} x\right| \leq\left|x^{T} \bar{C}_{i}^{T} \bar{C}_{i} x\right|+\left|x^{T} \mathcal{A}_{1}^{T} \bar{C}_{i}^{T} \bar{C}_{i} \mathcal{A}_{1} x\right|$. Hence, $\left\langle\nabla \delta_{i}\left(v_{i}\right), \bar{C}_{i}\left(\mathcal{A}_{1} x+\mathcal{B}_{1} e+\mathcal{D}_{1} \bar{\psi}(x)+\mathcal{E}_{1} w\right)\right\rangle \leq$ $\epsilon_{i}^{2}\left|x^{T}\left(\bar{C}_{i}^{T} \bar{C}_{i} \mathcal{A}_{1}+\mathcal{A}_{1}^{T} \bar{C}_{i}^{T} \bar{C}_{i}\right) x\right|+2 \epsilon_{i}^{2}\left(\mid x^{T} \bar{C}_{i}^{T} \bar{C}_{i}\left(\mathcal{B}_{1} e+\right.\right.$ $\left.\left.\mathcal{E}_{1} w\right)\left.|+L| \bar{C}_{i}^{T} \bar{C}_{i} \mathcal{D}_{1}|| x\right|^{2}\right) \leq L_{\delta_{i}} \delta_{i}+J_{i}(x, e, w)$, where $L_{\delta_{i}}=1$ and $J_{i}(x, e, w)=\epsilon_{i}^{2}\left(\left|\mathcal{A}_{1}^{T} \bar{C}_{i}^{T} \bar{C}_{i} \mathcal{A}_{1}\right|+2 L\left|\bar{C}_{i}^{T} \bar{C}_{i} \mathcal{D}_{1}\right|\right)|x|^{2}+$ $2 \epsilon_{i}^{2}\left|x^{T} \bar{C}_{i}^{T} \bar{C}_{i}\left(\mathcal{B}_{1} e+\mathcal{E}_{1} w\right)\right|$, hence item (iii) of Assumption 2 holds.

Let $V(x)=x^{T} P x$. Item (i) of Assumption 2 holds with $\underline{\alpha}_{V}(s):=\lambda_{\min }(P) s^{2}, \bar{\alpha}_{V}(s):=\lambda_{\max }(P) s^{2}$ for all $s \geq 0$. Note that, for all $x \in \mathbb{R}^{n_{x}}$ and almost all $e \in \mathbb{R}^{n_{e}}$, $\left\langle\nabla V(x), \mathcal{A}_{1} x+\mathcal{B}_{1} e+\mathcal{D}_{1} \bar{\psi}(x)+\mathcal{E}_{1} w\right\rangle=x^{T}\left(\mathcal{A}_{1}^{T} P+P \mathcal{A}_{1}\right) x+$ $2 x^{T} P \mathcal{B}_{1} e+2 L\left|\mathcal{D}_{1}\right| x^{T} P x+2 x^{T} P \mathcal{E}_{1} w$. We now post- and premultiply LMI in (24) respectively by the state vector $(x, e, w)$ and its transpose, re-arrange its terms, and derive from (24) and the definitions of $\mathcal{A}_{2}, \mathcal{B}_{2}$ and $\mathcal{E}_{2}$ defined after (22) that item (ii) of Assumption 2 holds with functions $\widetilde{H}_{i}, J_{i}, \delta_{i}, \varrho_{V, i}$ and $\gamma_{i}>0$ given in Proposition 1.

\section{E. Proof of Lemma 1}

The proof agrees with the one to Proposition 1 with replacing $\psi(x)$ by $\psi(y, e)$, and the property $|\bar{\psi}(y, e)| \leq L|e|$ in view of properties of $\psi$. Since $2 L|P x|\left|\mathcal{D}_{1}\right||e| \leq a_{V}|x|^{2}+$ $1 / a_{V}\left(L|P|\left|\mathcal{D}_{1} \| e\right|\right)^{2}$ for all $x \in \mathbb{R}^{n_{x}}, e \in \mathbb{R}^{n_{e}}$ and any $a_{V}>0$, and hence, $\left\langle\nabla V(x), \mathcal{A}_{1} x+\mathcal{B}_{1} e+\mathcal{D}_{1} \bar{\psi}(y, e)+\mathcal{E}_{1} w\right\rangle=$ $x^{T}\left(\mathcal{A}_{1}^{T} P+P \mathcal{A}_{1}\right) x+2 x^{T} P \mathcal{B}_{1} e+2 L|P x|\left|\mathcal{D}_{1} e\right|+2 x^{T} P \mathcal{E}_{1} w$. We then follow similar lines as in the proof of Proposition 1 to show Assumptions 1-2 hold. On the other hand, (24) always has a solution in this case since $\mathcal{A}_{1}$ is Hurwitz, which ensures $\Sigma_{11}<0$ and (24) follows by taking sufficiently large $\tilde{\theta}, \nu$ and small enough $\epsilon>0$.

\section{APPENDIX B TECHNICAL LEMMAS}

The next statements corresponds to Lemma II.1 in [34].

Lemma 2: Consider two functions $U_{1}: \mathbb{R}^{n} \rightarrow \mathbb{R}$ and $U_{2}: \mathbb{R}^{n} \rightarrow \mathbb{R}$ that have well-defined Clarke derivatives for all $x \in \mathbb{R}^{n}$ and $v \in \mathbb{R}^{n}$. Introduce three sets $A:=\{x$ : $\left.U_{1}(x)>U_{2}(x)\right\}, B:=\left\{x: U_{1}(x)<U_{2}(x)\right\}, \Gamma:=$ $\left\{x: U_{1}(x)=U_{2}(x)\right\}$. Then, for any $v \in \mathbb{R}^{n}$, the function $U(x):=\max \left\{U_{1}(x), U_{2}(x)\right\}$ satisfies $U^{\circ}(x ; v)=U_{1}^{\circ}(x ; v)$ for all $x \in A, U^{\circ}(x ; v)=U_{2}^{\circ}(x ; v)$ for all $x \in B$, and $U^{\circ}(x ; v) \leq \max \left\{U_{1}^{\circ}(x ; v), U_{2}^{\circ}(x ; v)\right\}$ for all $x \in \Gamma$.

Lemma 3: Let $i \in \bar{N}, \lambda_{i} \in\left[0, \lambda_{i}^{*}\right)$ and $T_{i}<T_{\mathrm{MASP}, i}\left(\lambda_{i}\right)$ with $\lambda_{i}^{*}$ and $T_{\mathrm{MASP}, i}\left(\lambda_{i}\right)$ defined in (14) and (15), respectively. Let $\bar{\mu}_{i}^{*}:=1 / \bar{\rho}_{i}, \underline{\mu}_{i}^{*}=\bar{\rho}_{i}$ with $\bar{\rho}_{i}$ defined after (13). There exist $\bar{\mu}_{i}>\underline{\mu}_{i}>0$, satisfying $0<\underline{\mu}_{i}^{*}<\underline{\mu}_{i}<\bar{\mu}_{i}<\bar{\mu}_{i}^{*}$, and $\tilde{\nu}_{i}>0$ such that the solution $\phi_{i}$ to $\dot{\phi}_{i}=-\left(2 L_{W_{i}}+\tilde{\nu}_{i}\right) \phi_{i}-\gamma_{i}\left(\phi_{i}^{2}+\right.$ 1), $\phi_{i}(0)=\bar{\mu}_{i}$, verifies $\phi_{i}(t) \in\left[\underline{\mu}_{i}, \bar{\mu}_{i}\right]^{i}$ for all $t \in\left[0, T_{i}\right]$, where $L_{W_{i}} \geq 0$ comes from Assumption 1 and $\gamma_{i}>0$ from Assumption 2. 
Proof of Lemma 3. Let $i \in \bar{N}, \lambda_{i} \in\left[0, \lambda_{i}^{*}\right)$ and $T_{i}<$ $T_{\text {MASP }, i}\left(\lambda_{i}\right)$. We first show that the following fact holds.

Fact 1: $0 \leq \frac{\gamma_{i} \lambda_{i}}{1-\lambda_{i} L_{\delta_{i}}}<1, i \in \bar{N}$.

Fact 1 holds since $0 \leq \frac{\gamma_{i} \lambda_{i}}{1-\lambda_{i} L_{\delta_{i}}} \leq \frac{\gamma_{i} \lambda_{i}}{1+\gamma_{i} \lambda_{i}}<1$ when $L_{\delta_{i}} \leq-\gamma_{i}$, and $\lambda_{i}<\frac{1}{L_{\delta_{i}}+\gamma_{i}}$ when $L_{\delta_{i}}>-\gamma_{i}$, in view of the definition of $\lambda_{i}^{*}$ in (14).

Fact 1 leads to $\bar{\rho}_{i}=\max \left\{\rho_{i}, \frac{\gamma_{i} \lambda_{i}}{1-\lambda_{i} L_{\delta_{i}}}\right\} \in[0,1)$ since $\rho_{i} \in[0,1)$. Hence, $0 \leq \underline{\mu}_{i}^{*}<\bar{\mu}_{i}^{*}$. Denote by $\mathcal{T}\left(\bar{\mu}_{i}, \underline{\mu}_{i}, \tilde{\nu}_{i}\right)$ the time it takes for the solution $\phi_{i}$ to decrease from $\bar{\mu}_{i}$ to $\mu_{i}$ for a given $\tilde{\nu}_{i} \geq 0$. In view of the dynamics of $\phi_{i}$, the function $\mathcal{T}_{i}$ is continuous in all its arguments, increasing in $\bar{\mu}_{i}$, decreasing in $\mu_{i}$ and decreasing in $\tilde{\nu}_{i}$. By following similar lines as in the proof of Lemma 2 in [28], we have that $T_{\mathrm{MASP}, i}$ defined in (15) satisfies $T_{\mathrm{MASP}, i}\left(\lambda_{i}\right)=\mathcal{T}_{i}\left(\lambda_{i}, \bar{\mu}_{i}^{*}, \mu_{i}^{*}, 0\right)$. Since $T_{i}<T_{\mathrm{MASP}, i}\left(\lambda_{i}\right)$, by continuity of $\mathcal{T}_{i}$ and in view of its increasing/decreasing properties, there exists a triplet $\left(\bar{\mu}_{i}, \underline{\mu}_{i}, \tilde{\nu}_{i}\right)$ with $\bar{\mu}_{i}<\bar{\mu}_{i}^{*}, \underline{\mu}_{i}>\underline{\mu}_{i}^{*}$ and $\tilde{\nu}_{i}>0$, such that $T_{i}=\mathcal{T}_{i}\left(\lambda_{i}, \bar{\mu}_{i}, \underline{\mu}_{i}, \tilde{\nu}_{i}\right)$ and $\phi_{i}(t) \in\left[\underline{\mu}_{i}, \bar{\mu}_{i}\right]$ holds for all $t \in\left[0, T_{i}\right]$.

Lemma 4: For any $\alpha_{1}, \ldots, \alpha_{N} \in \mathcal{K}, \underline{\alpha}\left(\sum_{i=1}^{N} s_{i}\right) \leq$ $\sum_{i=1}^{N} \alpha_{i}\left(s_{i}\right) \leq \bar{\alpha}\left(\sum_{i=1}^{N} s_{i}\right)$ holds for all $s_{i} \geq 0$ and $i \in \bar{N}$, where $\underline{\alpha}, \bar{\alpha}: s \mapsto \min \left\{\alpha_{1}\left(\frac{s}{N}\right), \ldots, \alpha_{N}\left(\frac{s}{N}\right)\right\} \in \mathcal{K}$ and $\bar{\alpha}:=\sum_{i=1}^{N} \alpha_{i}$.

Proof of Lemma 4. Let $\alpha_{1}, \ldots, \alpha_{N} \in \mathcal{K}$ and $s_{1}, \ldots, s_{N} \geq 0$. Since $\alpha_{1}, \ldots, \alpha_{N}$ are increasing functions, $\sum_{i=1}^{N} \alpha_{i}\left(s_{i}\right) \leq$ $\sum_{i=1}^{N} \alpha_{i}\left(s_{1}+\ldots, s_{N}\right)=\bar{\alpha}\left(\sum_{i=1}^{N} s_{i}\right)$. Without loss of generality, we assume that $s_{j}=\max \left\{s_{1}, \ldots, s_{N}\right\}$ for some $j \in \bar{N}$. It then follows that $\frac{1}{N} \sum_{i=1}^{N} s_{i} \leq$ $s_{j}$ and $\sum_{i=1}^{N} \alpha_{i}\left(s_{i}\right) \geq \alpha_{j}\left(s_{j}\right) \geq \alpha_{j}\left(\frac{1}{N} \sum_{i=1}^{N} s_{i}\right) \geq$ $\min \left\{\alpha_{1}\left(\frac{1}{N} \sum_{i=1}^{N} s_{i}\right), \ldots, \alpha_{N}\left(\frac{1}{N} \sum_{i=1}^{N} s_{i}\right)\right\}$, which completes the proof.

The proof of Lemma 5 follows the proof to Lemma 3 and is therefore is omitted.

Lemma 5: Let $\lambda \in\left[0, \lambda^{*}\right)$ and $T<T_{\mathrm{MASP}}(\lambda)$ with $\lambda^{*}$ and $T_{\mathrm{MASP}}(\lambda)$ defined as (17) and (18), respectively. There exist $\bar{\mu}>\mu>0$, satisfying $0<\bar{\rho}<\mu<\bar{\mu}<1 / \bar{\rho}$, and $\tilde{\nu} \in\left(0, a_{V}\right)$ such that the solution $\phi$ to $\dot{\phi}=-\left(2 L_{W}+\tilde{\nu}\right) \phi-$ $\gamma\left(\frac{L_{V}^{2}}{a_{V}-\tilde{\nu}} \phi^{2}+1\right), \phi(0)=\bar{\mu}$, verifies $\phi(t) \in[\underline{\mu}, \bar{\mu}]$ for all $t \in[0, T]$, where $\bar{\rho}:=\max \left\{\rho, \frac{\gamma \lambda}{a_{V}}\right\}, \rho \in[0,1) L_{W}, L_{V} \geq 0$ and $a_{V}, \gamma>0$ come from Assumption 3.

\section{REFERENCES}

[1] G. Walsh and H. Ye, "Scheduling of networked control systems," IEEE Control Systems Magzine, vol. 21, pp. 57-65, 2001.

[2] W. Heemels, K. Johansson, and P. Tabuada, "An introduction to eventtriggered and self-triggered control," in Proceedings of the IEEE Conference on Decision and Control, Hawaii, USA, 2012, pp. 3270-3285.

[3] P. Tabuada, "Event-triggered real-time scheduling of stabilizing control tasks," IEEE Transactions on Automatic Control, vol. 52, pp. 1680-1685, 2007.

[4] V. Dolk, D. Borgers, and W. Heemels, "Output-based and decentralized dynamic event-triggered control with guaranteed $L_{p}$-gain performance and Zeno-freeness," IEEE Transactions on Automatic Control, vol. 62, pp. 34-49, 2017.

[5] J. Lunze and D. Lehmann, "A state-feedback approach to event-based control," Automatica, vol. 46, pp. 211-215, 2010.

[6] X. Wang and M. Lemmon, "Event-triggering in distributed networked control systems," IEEE Transactions on Automatic Control, vol. 56, pp. 586-601, 2011.

[7] R. Postoyan, P. Tabuada, D. Nešić, and A. Anta, "A framework for the event-triggered stabilization of nonlinear systems," IEEE Transactions on Automatic Control, vol. 60, pp. 982-996, 2015.

[8] W. Heemels, M. Donkers, and A. Teel, "Periodic event-triggered control for linear systems," IEEE Transactions on Automatic Control, vol. 58, pp. 847-861, 2013.

[9] W. Heemels, R. Postoyan, M. Donkers, A. Teel, A. Anta, P. Tabuada, and D. Nešić, "Periodic event-triggered control," in Event-based control and signal processing, 2015, pp. 105-119.

[10] M. Abdelrahim, R. Postoyan, J. Daafouz, and D. Nešić, "Stabilization of nonlinear systems using event-triggered output feedback controllers," IEEE Transactions on Automatic Control, vol. 61, pp. 2682-2687, 2016.

[11] M. Abdelrahim, R. Postoyan, J. Daafouz, and D. Nešić, "Robust eventtriggered output feedback controllers for nonlinear systems," Automatica, vol. 75, pp. 96-108, 2017.

[12] R. Sanfelice and A. Teel, "Lyapunov analysis of sampled-and-hold hybrid feedbacks," in Proceedings of the 45th IEEE Conference on Decision and Control, San Diego, USA, 2006, pp. 4879-4884.

[13] X. Chen and F. Hao, "Periodic event-triggered state-feedback and outputfeedback control for linear systems," International Journal of Control, Automation and Systems, vol. 13, pp. 779-787, 2015.

[14] W. Heemels, G. Dullerud, and A. Teel, " $\mathcal{L}_{2}$-gain analysis for a class of hybrid systems with applications to reset and event-triggered control: A lifting approach," IEEE Transactions on Automatic Control, vol. 61, pp. 2766-2781, 2016

[15] A. Selivanov and E. Fridman, "Event-triggered $H_{\infty}$ control: a switching approach," IEEE Transactions on Automatic Control, vol. 61, pp. 32213226, 2016.

[16] M. Braksmayer and L. Mirkin, "Redesign of stabilizing discrete-time controllers to accommodate intermittent sampling," in Proceedings of the 2017 IFAC World Congress, Toulouse, France, 2017, pp. 2633-2638.

[17] D. P. Borgers, R. Postoyan, A. Anta, P. Tabuada, D. Nešić, and W. Heemels, "Periodic event-triggered control of nonlinear systems using overapproximation techniques," Automatica, vol. 94, pp. 81-87, 2018.

[18] L. Etienne, S. D. Gennaro, and J. Barbot, "Periodic event-triggered observation and control for nonlinear Lipschitz systems using impulsive observers," international Journal of Robust and Nonlinear Control, 2017. [Online]. Available: http://dx.doi.org/10.1002/rnc.3802

[19] E. A. Escolástico, M. Abdelrahim, M. Guinaldo, S. Dormido, and W. Heemels, "Design of periodic event-triggered control for polynomial systems: A delay system approach," in Proceedings of the 2017 IFAC World Congress, Toulouse, France, 2017, pp. 7887-7892.

[20] D. Nešić and A. Teel, "Input-output stability properties of networked control systems," IEEE Transactions on Automatic Control, vol. 49, pp. 1650-1667, 2004.

[21] C. Cai and A. R. Teel, "Characterizations of input-to-state stability for hybrid systems," Systems \& Control Letters, vol. 58, pp. 47-53, 2009.

[22] R. Goebel, R. Sanfelice, and A. Teel, Hybrid dynamical systems: Modeling, Stability, and Robustness. Princeton University Press, 2012.

[23] W. Wang, R. Postoyan, D. Nešić, and W. Heemels, "Stabilization of nonlinear systems using state-feedback periodic event-triggered controllers," in Proceedings of the 55th IEEE Conference on Decision and Control, Las Vegas, NV, USA, 2016, pp. 6808-6813. 
[24] — - "Periodic event-triggered output feedback control of nonlinear systems," in Proceedings of the 57th IEEE Conference on Decision and Control, Florida, USA, 2018, pp. 957-962.

[25] D. Nešić and A. Teel, "Input-to-state stability of networked control systems," Automatica, vol. 40, pp. 2121-2128, 2004.

[26] W. Heemels, A. Teel, N. van de Wouw, and D. Nešić, "Networked control systems with communication constraints: Tradeoffs between transmission intervals, delays and performance," IEEE Transactions on Automatic Control, vol. 55, pp. 1781-1796, 2010.

[27] D. Nešić, A. Teel, and D. Carnevale, "Explicit computation of the sampling period in emulation of controllers for nonlinear sampled-data systems," IEEE Transactions on Automatic Control, vol. 54, pp. 619624, 2009.

[28] D. Carnevale, A. Teel, and D. Nešić, "A Lyapunov proof of an improved maximum allowable transfer interval for networked control systems," IEEE Transactions on Automatic Control, vol. 52, pp. 892-897, 2007.

[29] M. Tabbara and D. Nešić, "Input-output stability with input-to-state stable protocols for quantized and networked control systems," in Proceedings of the 47th IEEE Conference on Decision and Control, Cancun, Mexico, 2008, pp. 2680-2685.

[30] M. Abdelrahim, R. Postoyan, J. Daafouz, D. Nešić, and W. Heemels, "Co-design of output feedback laws and event-triggering conditions for the $\mathcal{L}_{2}$-stabilization linear systems," Automatica, vol. 87, pp. 337-344, 2018.

[31] H. Khalil, Nonlinear Systems. Prentice Hall, Englewood Cliffs, NJ, 2002.

[32] A. Teel and L. Praly, "On assigning the derivative of a disturbance attenuation control Lyapunov function," Mathematics Control Signals Systems, vol. 13, pp. 95-124, 2000.

[33] Z. P. Jang, A. R. Teel, and L. Praly, "Small-gain theorem for ISS systems and applications," Mathematics Control Signals Systems, vol. 7, pp. 95120, 1994.

[34] D. Liberzon, D. Nešić, and A. Teel, "Lyapunov-based small-gain theorems for hybrid systems," IEEE Transactions on Automatic Control, vol. 59, pp. 1395-1410, 2014.

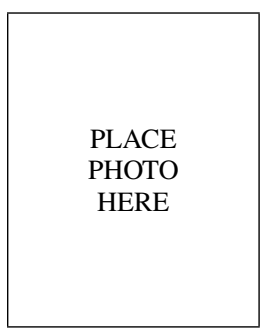

Wei Wang received her B.E. and M.E. degrees in Mechanical Engineering from Qingdao University of Science and Technology in 1994 and 2002, respectively. She obtained her Ph.D degree in Electrical Engineering at the University of Melbourne, Australia, in 2011. Since July 2011, she is a postdoctoral researcher in the Department of Electrical and Electronic Engineering at the University of Melbourne. Her research interests include nonlinear control systems, hybrid systems and networked control systems.

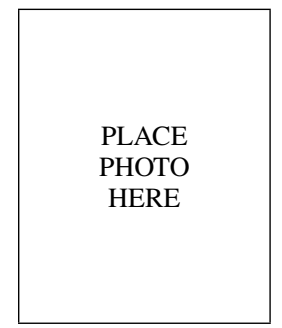

Romain Postoyan received the M.Sc. degree in Electrical and Control Engineering from ENSEEIHT (France) in 2005. He obtained the M.Sc. by Research in Control Theory \& Application from Coventry University (United Kingdom) in 2006 and the Ph.D. in Control Theory from Université ParisSud (France) in 2009. In 2010, he was a research assistant at the University of Melbourne (Australia). Since 2011, he is a CNRS researcher at the Centre de Recherche en Automatique de Nancy (France). He serves/served as an associate editor for the journals: Automatica, IEEE Control Systems Letters and IMA Journal of Mathematical Control and Information.

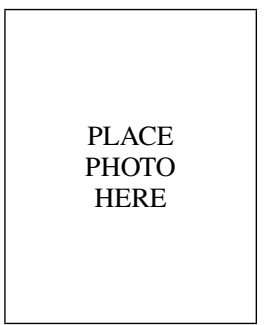

Dragan Nešić is a Professor in the Department of Electrical and Electronic Engineering (DEEE) at The University of Melbourne, Australia. He received his BE degree in Mechanical Engineering from The University of Belgrade, Yugoslavia in 1990, and his Ph.D. degree from Systems Engineering, RSISE, Australian National University, Canberra, Australia in 1997. Since February 1999 he has been with The University of Melbourne. His research interests include networked control systems, discrete-time, sampled-data and continuous-time nonlinear control systems, input-to-state stability, extremum seeking control, applications of symbolic computation in control theory, hybrid control systems, and so on. $\mathrm{He}$ was awarded a Humboldt Research Fellowship (2003) by the Alexander von Humboldt Foundation, an Australian Professorial Fellowship (2004-2009) and Future Fellowship (2010-2014) by the Australian Research Council. He was a Distinguished Lecturer of CSS, IEEE. He is a Fellow of IEEE and a Fellow of IEAust. He served as an Associate Editor for the journals Automatica, IEEE Transactions on Automatic Control, IEEE Transactions on Control of Networked Systems, Systems and Control Letters and European Journal of Control.

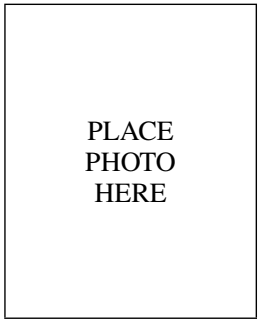

W. P. M. H. Heemels received the M.Sc. degree in mathematics and the Ph.D. degree in control theory (both summa cum laude) from the Eindhoven University of Technology (TU/e), Eindhoven, The Netherlands, in 1995 and 1999, respectively. From 2000 to 2004, he was with the Electrical Engineering Department, TU/e, as an Assistant Professor and from 2004 to 2006 with the Embedded Systems Institute (ESI) as a Research Fellow. Since 2006, he has been with the Department of Mechanical Engineering, TU/e, where he is currently a Full Professor in the Control Systems Technology Group. He held visiting research positions at the Swiss Federal Institute of Technology (ETH), Zurich, Switzerland (2001) and at the University of California at Santa Barbara (2008). In 2004 he was also at the Research and Development Laboratory, Océ, Venlo, The Netherlands. His current research interests include hybrid and cyber-physical systems, networked and event-triggered control systems, and constrained systems including model predictive control. Dr. Heemels served/serves on the editorial boards of Automatica, Nonlinear Analysis: Hybrid Systems, Annual Reviews in Control, and the IEEE TRANSACTIONS ON AUTOMATIC CONTROL. He was a recipient of a personal VICI grant awarded by NWO (The Netherlands Organization for Scientific Research) and STW (Dutch Technology Foundation) 


\section{University Library}

\section{- M M N E R VA A gateway to Melbourne's research publications}

Minerva Access is the Institutional Repository of The University of Melbourne

Author/s:

Wang, W;Postoyan, R;Nesic, D;Heemels, WPMH

Title:

Periodic Event-Triggered Control for Nonlinear Networked Control Systems

Date:

2020-02-01

Citation:

Wang, W., Postoyan, R., Nesic, D. \& Heemels, W. P. M. H. (2020). Periodic Event-Triggered Control for Nonlinear Networked Control Systems. IEEE TRANSACTIONS ON AUTOMATIC CONTROL, 65 (2), pp.620-635. https://doi.org/10.1109/TAC.2019.2914255.

Persistent Link:

http://hdl.handle.net/11343/249538 